\title{
Partitioned simulation of the interaction between an elastic structure and free surface flow
}

\author{
Joris Degroote $^{\mathrm{a}}$, Antonio Souto-Iglesias ${ }^{\mathrm{b}}$, Wim Van Paepegem ${ }^{\mathrm{c}}$, \\ Sebastiaan Annerel $^{\mathrm{a}}$, Peter Bruggeman ${ }^{\mathrm{d}}$, Jan Vierendeels ${ }^{*, \mathrm{a}}$ \\ ${ }^{a}$ Department of Flow, Heat and Combustion Mechanics, Ghent University, \\ Sint-Pietersnieuwstraat 41, B-9000 Ghent, Belgium \\ ${ }^{b}$ Naval Architecture Department (ETSIN), Technical University of Madrid (UPM), \\ Avda. Arco de la Victoria s/n, 28040 Madrid, Spain \\ ${ }^{c}$ Department of Materials Science and Engineering, Ghent University, \\ Sint-Pietersnieuwstraat 41, B-9000 Ghent, Belgium \\ ${ }^{d}$ Department of Applied Physics, Eindhoven University of Technology, \\ P.O.-Box 513, 5600 MB Eindhoven, The Netherlands
}

\begin{abstract}
Currently, the interaction between free surface flow and an elastic structure is simulated with monolithic codes which calculate the deformation of the structure and the liquid-gas flow simultaneously. In this work, this interaction is calculated in a partitioned way with a separate flow solver and a separate structural solver using the interface quasi-Newton algorithm with approximation for the inverse of the Jacobian from a least-squares model (IQN-ILS). The interaction between an elastic beam and a sloshing liquid in a rolling tank is calculated and the results agree well with experimental data. Subsequently, the impact of both a rigid cylinder and a flexible composite cylinder on a water surface is simulated to assess the effect of slamming on the components of certain wave-energy converters. The impact pressure on the bottom of the rigid cylinder is nearly twice as high as on the flexible cylinder, which emphasizes the need for fluid-structure interaction

\footnotetext{
${ }^{*}$ Corresponding author

Email addresses: Joris. Degroote@UGent . be (Joris Degroote), Antonio. Souto@upm. es (Antonio Souto-Iglesias), Wim. VanPaepegem@UGent . be (Wim Van Paepegem), Sebastiaan. Annerel@UGent. be (Sebastiaan Annerel), p.j.bruggeman@tue.nl (Peter Bruggeman), Jan.Vierendeels@UGent . be (Jan Vierendeels)

URL: http: / /www.FSI. UGent . be/ (Joris Degroote), http://www. composites. UGent.be/ (Wim Van Paepegem)
} 
calculations in the design process of these wave-energy converters. For both the rolling tank simulations and the impact simulations, grid refinement is performed and the IQN-ILS algorithm requires the same number of iterations on each grid. The simulations on the coarse grid are also executed using Gauss-Seidel coupling iterations with Aitken relaxation which requires significantly more coupling iterations per time step.

Key words:

Fluid-structure interaction, Partitioned, Free surface, Volume-of-Fluid, Rolling tank, Impact, IQN-ILS, Aitken relaxation

\section{Introduction}

Over the past decade, the simulation of fluid-structure interaction (FSI) has gained interest, resulting in numerous biomedical $[1,2,3]$ and engineering $[4$, 5] applications. More recently, the level of complexity of FSI simulations has increased by the addition of advanced models such as free surface flow to the coupled problem $[6,7,8,9,10]$. Multiphase flow can be highly unsteady because of waves and droplets. The interaction between such an unsteady flow and a structure can change abruptly due to impact of a structure on a free surface or a wave hitting an already deforming structure. This strong time dependence causes additional difficulties in FSI simulations.

Free surface flow has since long fascinated scientists and engineers, possibly due to the countless spectacular applications, and several numerical methods have been devised. Most of these methods can be categorized as interface-tracking, interface-capturing or particle methods. Interface-tracking methods represent the liquid-gas interface by means of a chain of grid nodes in $2 \mathrm{D}$ or a surface in 3D. These grid nodes move at the same speed as the fluids over a static [11] or deforming $[12,13]$ fluid grid. Interface-capturing methods use a grid which does not deform due to the motion of the fluid and some kind of marker which is transported with the flow to determine on which side of the liquid-gas interface a cell is located. The Volume-Of-Fluid (VOF) method employs a marker variable to store the fraction of the cell that is filled with a given phase $[14,15]$ and the Level Set method indicates the liquid-gas interface with the zero level of a smooth function [16]. The Particle Finite Element Method (PFEM) [17] and Smoothed Particle Hydrodynamics (SPH) [18] are particle methods but also lattice methods [19] fit in this category. Several benchmarks have been established to compare and verify all these simulation techniques, for example the well-known dam-break problem 
[20].

FSI and coupled problems in general can be simulated in either a monolithic or a partitioned way. In the monolithic approach, the equations of the subproblems are solved simultaneously, thereby taking into account the interaction between the subproblems during the solution process. This results in a large system of generally nonlinear coupled equations which is often solved with Newton iterations [21] with suitable preconditioning for the different blocks in the resulting linear systems. In a partitioned simulation, however, the equations of each subproblem are solved separately with a code that has been developed specifically for that kind of equations [22]. A coupling algorithm incorporates the interaction between the subproblems, often by performing iterations between the subproblems. Algorithms without coupling iterations [23] and Gauss-Seidel iterations [1, 24, 25] are mostly unstable in the case of strong interaction between the flow and the structure. However, quasi-Newton iterations [26, 27] or Newton-Krylov techniques $[28,29]$ can be used to solve such FSI problems in a partitioned way, even with black-box solvers. The main advantage of monolithic simulations is the stability of the solution process, whereas the most important benefit of the partitioned approach is that existing, mature and optimized codes for the subproblems can be reused.

Several simulations of FSI with a free surface have previously been performed with considerable attention for experimental validation. Walhorn et al. [6] use a space-time finite element discretization and the Level Set method to simulate a rising bubble and dam-break with an elastic obstacle. Antoci et al. [7] work with SPH to calculate a variation of the dam-break problem in which the dam does not disappear but becomes flexible at the bottom. Idelsohn et al. $[8,9]$ employ PFEM to simulate various cases, among which dam-break with a flexible obstacle, a solid object impacting and floating on water and the interaction between a flexible structure and the sloshing flow in a rolling tank. Potapov et al. [10] simulate fluid-structure interaction with tearing structures using SPH.

However, all results mentioned in the previous paragraph have been obtained using monolithic techniques. In this paper, it is demonstrated how partitioned simulation of the interaction between an elastic structure and free surface flow can be performed. A finite volume flow code which solves the Navier-Stokes equations in arbitrary Lagrangian-Eulerian (ALE) formulation with a VOF model for the free surface is coupled with a finite element structural code by means of interface quasi-Newton iterations using an approximation for the inverse of the Jacobian from a least-squares model (IQN-ILS) [27]. This coupling technique treats both the flow solver and the structural solver as a black box and the algorithm will be 
explained in detail below. The performance (measured as the average number of coupling iterations per time step) of the IQN-ILS coupling algorithm is compared with another partitioned algorithm, namely Gauss-Seidel iterations with Aitken relaxation [30, 31].

An existing case, namely the sloshing flow in a rolling tank with a flexible obstacle [9], is simulated and the results are compared with experiments to demonstrate that the partitioned approach results in the correct solution. Subsequently, the impact of both a rigid and a flexible composite cylinder on a water surface is simulated to accelerate the design process of a particular component for floating wave-energy converters.

Section 2 describes the governing equations for the flow and the structure and their discretization. The IQN-ILS coupling algorithm is explained in Section 3, followed by a brief description of Gauss-Seidel iterations with Aitken relaxation in Section 4. The simulations of the rolling tank and falling cylinder are presented in Section 5 and Section 6, respectively, and the conclusions are listed in Section 7.

\section{Governing equations}

In this section, the governing equations for the fluid flow and the structure and their discretization are outlined, followed by the equilibrium conditions on the fluid-structure interface. The subdomains are indicated as $\Omega_{f}$ and $\Omega_{s}$ and their boundaries as $\Gamma_{f}$ and $\Gamma_{s}$, with the subscript $f$ denoting fluid and $s$ solid. The fluidstructure interface $\Gamma_{i}=\Gamma_{f} \cap \Gamma_{s}$ is the common boundary of these subdomains (Figure 1).

\subsection{Flow equations}

The liquid and the gas in the free surface flow are both considered incompressible and mutually immiscible. This multiphase flow is modeled with the VOF technique, which introduces a scalar volume fraction $\alpha_{f}$ throughout the fluid domain to distinguish the liquid from the gas $[14,15]$. A region is filled with liquid only if the volume fraction is one and with gas only if the volume fraction is zero. The fluid properties such as the fluid density $\rho_{f}$ are written as a function of the volume fraction

$$
\rho_{f}=\alpha_{f} \rho_{l}+\left(1-\alpha_{f}\right) \rho_{g}
$$

with $\rho_{l}$ and $\rho_{g}$ the density of the liquid and the gas, respectively. Similarly for the fluid viscosity $\mu_{f}$. 
The unsteady, isothermal flow of the liquid and the gas is governed by the conservation of mass and a single set of Navier-Stokes equations, given by

$$
\begin{gathered}
\frac{\partial \rho_{f}}{\partial t}+\nabla \cdot\left(\rho_{f} \boldsymbol{v}_{f}\right)=0 \\
\frac{\partial \rho_{f} \boldsymbol{v}_{f}}{\partial t}+\nabla \cdot\left(\rho_{f} \boldsymbol{v}_{f} \boldsymbol{v}_{f}\right)-\nabla \cdot \boldsymbol{\tau}_{f}=\boldsymbol{f}_{f}
\end{gathered}
$$

for $\boldsymbol{x} \in \Omega_{f}$. The flow velocity is denoted by $\boldsymbol{v}_{f}$ and the time by $t . \boldsymbol{f}_{f}$ represents the body forces per unit of volume on the fluid. In this paper, gravity is the only body force so $\boldsymbol{f}_{f}=-\rho_{f} g \mathbf{1}_{y}$ with $g=9.81 \mathrm{~m} / \mathrm{s}^{2}$ the gravitational acceleration and $\mathbf{1}_{y}$ the unit vector in the vertical direction as indicated in Figure 1. For the Newtonian fluids under consideration, the stress tensor is defined as

$$
\boldsymbol{\tau}_{f}=-p \boldsymbol{I}+2 \mu \gamma
$$

with the rate of strain tensor $\gamma$ given by

$$
\boldsymbol{\gamma}=\frac{1}{2}\left[\nabla \boldsymbol{v}_{f}+\left(\nabla \boldsymbol{v}_{f}\right)^{\mathrm{T}}\right]
$$

For two incompressible fluid phases, the mass conservation of the phases results in an equation for the volume fraction, namely

$$
\frac{\partial \alpha_{f}}{\partial t}+\nabla \cdot\left(\alpha_{f} \boldsymbol{v}_{f}\right)=0
$$

There is no mass transfer between the liquid and the gas. Also, surface tension is not taken into account because both the Reynolds number

$$
R e=\frac{\rho_{l} v_{l} L}{\mu_{l}}
$$

and the Weber number

$$
W e=\frac{\rho_{l} v_{l} L}{\sigma_{l g}}
$$

are much larger than one, with $\sigma_{l g}$ being the surface tension coefficient between the liquid and the gas and $L$ the appropriate length scale.

The flow equations are discretized in space on a grid with triangular and rectangular cells using the finite volume method. Scalars are stored in the cell centres and a power law is used to obtain momentum variables at the faces. Gradients 
at the cell centres are calculated from the face values using the Green-Gauss theorem. The face values for the gradient calculations are the arithmetic average of the node values, which are in turn the weighted average of the values in the cells around the node. The pressure interpolation at the faces is performed with a staggered grid approach similar to the one described by Patankar [32]. Eq. (2) are solved using the Pressure-Implicit with Splitting of Operators (PISO) scheme with skewness and neighbour correction. Algebraic multigrid is employed to accelerate the convergence.

The grid of the fluid domain is deforming, driven by the deformation of the fluid-structure interface. Smoothing with fictitious springs between the grid nodes is applied for deformations during the time step. Cells which have either become too skewed or which fall outside the range of desired cell sizes are eliminated once in each time step. The implicit backward Euler time discretization of Eq. (2) in ALE formulation is first order accurate on a moving grid.

Eq. (4) for the volume fraction is solved with first order explicit time discretization but the time step for this equation is only a fraction of the time step of the FSI calculation such that the Courant number does not exceed 0.25 near the liquid-gas interface. However, the volume fraction is recalculated after each grid deformation and the convective flux coefficients are updated based on the new volume fractions. The liquid-gas interface is reconstructed with a piecewiselinear approach for an accurate calculation of the fluxes through the faces near the liquid-gas interface [33].

\subsection{Structural equations}

The deformation $\boldsymbol{d}_{s}$ of the structure is determined by the conservation of momentum

$$
\rho_{s} \frac{\partial^{2} \boldsymbol{d}_{s}}{\partial t^{2}}-\nabla \cdot \boldsymbol{\sigma}_{s}=\boldsymbol{f}_{s}
$$

for $\boldsymbol{x} \in \Omega_{s}$ with $\rho_{s}$ the structural density and $\boldsymbol{f}_{s}=-\rho_{s} g \mathbf{1}_{y}$ the body force per unit volume on the structure. The relation between the stress tensor $\sigma_{s}$ and the strain tensor

$$
\boldsymbol{\epsilon}_{s}=\frac{1}{2}\left[\nabla \boldsymbol{d}_{s}+\left(\nabla \boldsymbol{d}_{s}\right)^{T}\right]
$$

is given by the constitutive equation of the material, in this case a linear-elastic material law.

$$
\sigma_{s}=C: \epsilon_{s}
$$


The value of $\boldsymbol{C}$ depends on the material and will therefore be different for the test cases presented in Section 5 and Section 6, where this and other case-dependent assumptions will be documented.

The structure is discretized with finite elements. Geometric nonlinearity is taken into account during the solution process and the stress on the fluid-structure interface follows the rotation of the structure during the time step. Unconditionally stable implicit Hilber-Hughes-Taylor time integration [34] is used with a small numerical damping parameter $\alpha_{s}=-0.05$.

\subsection{Equilibrium conditions}

The equilibrium conditions on the fluid-structure interface are the kinematic condition

and the dynamic condition

$$
\boldsymbol{v}_{f}=\frac{\partial \boldsymbol{d}_{s}}{\partial t}
$$

$$
\boldsymbol{n}_{f} \cdot \boldsymbol{\sigma}_{f}=-\boldsymbol{n}_{s} \cdot \boldsymbol{\sigma}_{s}
$$

for $\boldsymbol{x} \in \Gamma_{i}$ with $\boldsymbol{d}$ the displacement, $\boldsymbol{\sigma}$ the stress tensor and $\boldsymbol{n}$ the unit normal vector that points outwards from the domain $\Omega$. The Dirichlet-Neumann formulation of the FSI problem is employed, which means that the flow equations are solved for a given velocity of the fluid-structure interface, whereas a stress is imposed on the fluid-structure boundary of the solid domain. The time discretization converts Eq. (9) into equality of the displacements on the fluid-structure interface. Appropriate conditions such as no-slip walls and constant pressure boundaries are imposed on $\Gamma_{f} \backslash \Gamma_{i}$ and displacements or rotations are applied on $\Gamma_{s} \backslash \Gamma_{i}$.

As the fluid and solid have a different discretization on the fluid-structure interface, an interpolation has to be performed. To transfer the displacement from the solid side to the fluid side of the interface, the fluid grid nodes are projected orthogonally on the boundary of the structural grid, after which the displacement at the location of this projection is calculated with linear interpolation of the values at the two nearest structural nodes. The stresses on the solid side of the fluid-structure interface are obtained in an analogous way from the stresses on the fluid side by orthogonal projection of the load integration points on the fluid grid followed by linear interpolation. Although other interpolation techniques exist $[35,36]$, this simple approach is chosen because it does not require any information about the connectivity or discretization in the solvers, which is consistent with the black-box approach of the IQN-ILS coupling algorithm and the GaussSeidel iterations with Aitken relaxation. The interpolation will be hidden in the following sections to avoid additional notation. 


\section{Interface quasi-Newton coupling algorithm}

In this section, the flow solver and the structural solver are redefined as functions with the degrees-of-freedom on the interface as input and output. These functions will subsequently be used in the explanation of the coupling algorithms. In the remainder of this paper, all values and functions are at the new time level $n+1$, unless indicated otherwise with a superscript $n$. A right superscript $k$ indicates the coupling iteration within time step $n+1$ and a subscript denotes the element in a vector. Capital letters denote matrices, bold lower case letters and lower case letters represent vectors and scalars, respectively.

The displacement degrees-of-freedom of all nodes on the fluid-structure interface are grouped in a vector $\boldsymbol{d} \in \mathbb{R}^{u}$ and the normal stress components $\boldsymbol{\sigma} \cdot \boldsymbol{n}$ on all faces of the interface are gathered in a vector $t \in \mathbb{R}^{w}$. The function

$$
t=\mathcal{F}(\boldsymbol{d})
$$

is referred to as the flow solver and it concisely represents several operations. The displacement of the fluid-structure interface is passed on to the flow code and the grid of the fluid domain adjacent to the interface is adapted accordingly. Subsequently, the grid velocity is calculated and the flow equations are solved for the fluid state in the entire fluid domain, which also results in a stress distribution on the interface.

The structural solver is represented by the function

$$
\boldsymbol{d}=\mathcal{S}(\boldsymbol{t})
$$

This expression indicates that the stress distribution on the interface is given to the structural code which then calculates the displacement of the entire structure and thus also the new displacement of the fluid-structure interface. It is important to notice that $\mathcal{F}$ and $\mathcal{S}$ both solve a problem in a subdomain while their input and output is limited to the fluid-structure interface. Operations on $\boldsymbol{d}$ and $\boldsymbol{t}$ are therefore fast compared to evaluations of these functions.

The equilibrium conditions in Section 2.3 have to be satisfied in each time step of the FSI simulations so Eq. (11) and Eq. (12) have to be statisfied by the same vector $\boldsymbol{d}$ and $\boldsymbol{t}$. Elimination of $\boldsymbol{t}$ results in a set of equations for the displacement vector only

$$
\mathcal{S} \circ \mathcal{F}(\boldsymbol{d})=\boldsymbol{d}
$$

which is subsequently reformulated as a nonlinear root-finding problem in the interface's displacement

$$
\boldsymbol{r}(\boldsymbol{d})=\mathcal{S} \circ \mathcal{F}(\boldsymbol{d})-\boldsymbol{d}=\mathbf{0} .
$$


The dependence of $\boldsymbol{r}$ on $\boldsymbol{d}$ is further often omitted for clarity. This nonlinear equation in $\boldsymbol{d}$ is solved with quasi-Newton iterations

$$
\begin{aligned}
& \widehat{\left.\frac{\mathrm{d} \boldsymbol{r}}{\mathrm{d} \boldsymbol{d}}\right|_{\boldsymbol{d}^{k}}} \Delta \boldsymbol{d}^{k}=-\boldsymbol{r}^{k} \\
& \boldsymbol{d}^{k+1}=\boldsymbol{d}^{k}+\Delta \boldsymbol{d}^{k}
\end{aligned}
$$

and a hat is used to indicate the approximation of the Jacobian. This approximation is necessary because the exact Jacobian of $\boldsymbol{r}(\boldsymbol{d})$ is unknown as the Jacobians of the black-box functions $\mathcal{F}$ and $\mathcal{S}$ are unavailable. In each quasi-Newton iteration, the residual vector is calculated as the output of the structural solver $\left(\tilde{\boldsymbol{d}}^{k+1}\right)$ minus the input of the flow solver $\left(\boldsymbol{d}^{k}\right)$

$$
\boldsymbol{r}^{k}=\boldsymbol{r}\left(\boldsymbol{d}^{k}\right)=\mathcal{S} \circ \mathcal{F}\left(\boldsymbol{d}^{k}\right)-\boldsymbol{d}^{k}=\tilde{\boldsymbol{d}}^{k+1}-\boldsymbol{d}^{k}
$$

A tilde indicates that the displacement has been calculated by the structural solver to distinguish it from the displacement given to the flow solver. Since the displacement calculated by $\mathcal{S}$ is only an intermediate value that is not used in the next coupling iteration, the tilde is dropped once the displacement for the next iteration has been calculated.

If the Jacobian $\mathrm{d} \boldsymbol{r} / \mathrm{d} \boldsymbol{d}$ is approximated and quasi-Newton iterations are performed, black-box solvers can be used. However, the linear system Eq. (15a) with as dimension the number of degrees-of-freedom in the interface's displacement has to be solved in each quasi-Newton iteration. Although the number of degreesof-freedom in the interface's displacement is generally smaller than the number of degrees-of-freedom in the entire fluid and structure domain, the Jacobian matrix $\mathrm{d} \boldsymbol{r} / \mathrm{d} \boldsymbol{d}$ is usually dense. As a result, the solution of the linear system Eq. (15a) corresponds to a significant computational cost in large simulations, especially if a direct solver is used. It is therefore more advantageous to approximate the inverse of the Jacobian by applying the least-squares technique introduced by Vierendeels et al. [26] on a particular set of vectors, as will be explained below. This technique can also be used to solve linear systems as demonstrated in [37].

By approximating the inverse of the Jacobian, the quasi-Newton iterations Eq. (15) can be written as

$$
\boldsymbol{d}^{k+1}=\boldsymbol{d}^{k}+\left(\widehat{\left.\mathrm{d} \boldsymbol{r}\right|_{\boldsymbol{d}^{k}}}\right)^{-1}\left(-\boldsymbol{r}^{k}\right)
$$


It can be seen from Eq. (17) that the approximation for the inverse of the Jacobian does not have to be created explicitly; a procedure to calculate the product of this matrix with the vector $-\boldsymbol{r}^{k}$ is sufficient. The vector $-\boldsymbol{r}^{k}$ is the difference between the desired residual, i.e. $\mathbf{0}$, and the current residual $\boldsymbol{r}^{k}$ and it is further denoted as $\Delta \boldsymbol{r}=\mathbf{0}-\boldsymbol{r}^{k}$. The correction of the displacement in Eq. (17) is rewritten as

$$
\Delta \boldsymbol{d}^{k}=\widehat{\left(\frac{\mathrm{d} \boldsymbol{r}}{\mathrm{d} \boldsymbol{d}}\right)^{-1}}\left(-\boldsymbol{r}^{k}\right) \approx \frac{\widehat{\mathrm{d} \boldsymbol{d}}}{\mathrm{d} \boldsymbol{r}}\left(-\boldsymbol{r}^{k}\right)
$$

with a slight abuse of notation. After substitution of the definition of the residual $\boldsymbol{r}=\tilde{\boldsymbol{d}}-\boldsymbol{d}$, this becomes

$$
\begin{aligned}
\Delta \boldsymbol{d}^{k} & \approx \frac{\widehat{\mathrm{d} \boldsymbol{d}}}{\mathrm{d} \boldsymbol{r}}\left(-\boldsymbol{r}^{k}\right) \\
& =\left(\frac{\widehat{\mathrm{d} \tilde{\boldsymbol{d}}}}{\mathrm{d} \boldsymbol{r}}-\boldsymbol{I}\right)\left(-\boldsymbol{r}^{k}\right) \\
& =\frac{\widehat{\mathrm{d} \tilde{\boldsymbol{d}}}}{\mathrm{d} \boldsymbol{r}}\left(-\boldsymbol{r}^{k}\right)+\boldsymbol{r}^{k}
\end{aligned}
$$

Eq. (19c) indicates that the change $\Delta \tilde{\boldsymbol{d}}$ of the structural solver's output due to a given change of the residual $\Delta \boldsymbol{r}=-\boldsymbol{r}^{k}$

$$
\Delta \tilde{\boldsymbol{d}}=\frac{\widehat{\mathrm{d} \tilde{\boldsymbol{d}}}}{\mathrm{d} \boldsymbol{r}} \cdot\left(-\boldsymbol{r}^{k}\right)
$$

has to be approximated. This is done with data obtained during the previous quasiNewton iterations: Eq. (16) shows that the flow equations and structural equations are solved in quasi-Newton iteration $k$, resulting in $\tilde{\boldsymbol{d}}^{k+1}=\mathcal{S} \circ \mathcal{F}\left(\boldsymbol{d}^{k}\right)$ and the corresponding residual $\boldsymbol{r}^{k}$. To predict how $\tilde{\boldsymbol{d}}$ changes when $\boldsymbol{r}$ changes, these vectors are converted into differences with respect to the first quasi-Newton iteration.

$$
\begin{gathered}
\Delta \boldsymbol{r}^{k}=\boldsymbol{r}^{k}-\boldsymbol{r}^{0} \\
\Delta \tilde{\boldsymbol{d}}^{k+1}=\tilde{\boldsymbol{d}}^{k+1}-\tilde{\boldsymbol{d}}^{1}
\end{gathered}
$$

Each quasi-Newton iteration generates an additional vector $\Delta \boldsymbol{r}$ and the corresponding vector $\Delta \tilde{\boldsymbol{d}}$. These vectors are stored as the columns of the matrices

$$
\boldsymbol{V}^{k}=\left[\begin{array}{lllll}
\Delta \boldsymbol{r}^{k-1} & \Delta \boldsymbol{r}^{k-2} & \ldots & \Delta \boldsymbol{r}^{1} & \Delta \boldsymbol{r}^{0}
\end{array}\right]
$$


and

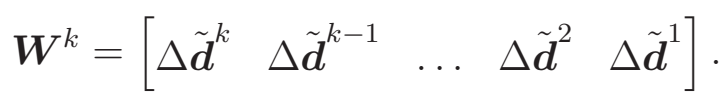

The number of columns in $\boldsymbol{V}^{k}$ and $\boldsymbol{W}^{k}$ is indicated as $v$ and it is generally much smaller than the number of rows $u$. Nevertheless, in simulations with a low number of degrees-of-freedom on the interface, it is possible that the number of columns has to be limited to $u$ by discarding the rightmost columns.

The desired change of the residual $\Delta \boldsymbol{r}=\mathbf{0}-\boldsymbol{r}^{k}$ is approximated as a linear combination of the known $\Delta \boldsymbol{r}^{i}$

$$
\Delta \boldsymbol{r} \approx \boldsymbol{V}^{k} \boldsymbol{c}^{k}
$$

with $c^{k} \in \mathbb{R}^{v}$ the coefficients of the decomposition. Because $v \leq u$, Eq. (23) is an overdetermined set of equations for the elements of $\boldsymbol{c}^{k}$ and hence the least-squares solution to this linear system is calculated. Therefore, the so-called economy size QR-decomposition of $\boldsymbol{V}^{k}$ is calculated using Householder transformations [38]

$$
\boldsymbol{V}^{k}=\boldsymbol{Q}^{k} \boldsymbol{R}^{k}
$$

with $\boldsymbol{Q}^{k} \in \mathbb{R}^{u \times v}$ an orthogonal matrix and $\boldsymbol{R}^{k} \in \mathbb{R}^{v \times v}$ an upper triangular matrix. The coefficient vector $\boldsymbol{c}^{k}$ is then determined by solving the triangular system

$$
\boldsymbol{R}^{k} \boldsymbol{c}^{k}=\boldsymbol{Q}^{k^{\mathrm{T}}} \Delta \boldsymbol{r}
$$

using back substitution. If a $\Delta \boldsymbol{r}^{i}$ vector is (almost) a linear combination of other $\Delta \boldsymbol{r}^{j}$ vectors, one of the diagonal elements of $\boldsymbol{R}^{k}$ will (almost) be zero. Consequently, the equation corresponding to that row of $\boldsymbol{R}^{k}$ cannot be solved during the back substitution. If a small diagonal element is detected, the corresponding column in $\boldsymbol{V}^{k}$ is removed and the QR-decomposition (Eq. (24)) and the solution of the triangular system (Eq. (25)) are repeated until none of the diagonal elements is too small. The tolerance for the detection of small diagonal elements depends on how accurately the flow equations and structural equations are solved.

The $\Delta \tilde{\boldsymbol{d}}$ that corresponds to $\Delta \boldsymbol{r} \approx \boldsymbol{V}^{k} \boldsymbol{c}^{k}$ can be approximated using the same decomposition coefficients $\boldsymbol{c}^{k}$ but with respect to $\boldsymbol{W}^{k}$ because there is a one-toone relation between the columns of $\boldsymbol{V}^{k}$ and $\boldsymbol{W}^{k}$. Consequently, the $\Delta \tilde{\boldsymbol{d}}$ sought after in Eq. (20) is given by

$$
\Delta \tilde{\boldsymbol{d}}=\boldsymbol{W}^{k} \boldsymbol{c}^{k} .
$$

Substitution of Eq. (26) in Eq. (19c) yields

$$
\Delta \boldsymbol{d}=\boldsymbol{W}^{k} \boldsymbol{c}^{k}+\boldsymbol{r}^{k}
$$


The complete IQN-ILS technique is shown in the algorithm below. Because the matrices $\boldsymbol{V}^{k}$ and $\boldsymbol{W}^{k}$ have to contain at least one column, a relaxation with factor $\omega$ (line 6) is performed in the second coupling iteration of each time step. The quasi-Newton iterations start from the initial guess

$$
\boldsymbol{d}^{n+1,0}=\frac{5}{2} \boldsymbol{d}^{n}-2 \boldsymbol{d}^{n-1}+\frac{1}{2} \boldsymbol{d}^{n-2}
$$

which is an extrapolation based on the previous time steps. Lower order extrapolations are used for the first two time steps. The iterations in the time step have converged when $\left\|\boldsymbol{r}^{k}\right\|_{2} \leq \epsilon_{o}$ with $\epsilon_{o}$ the convergence tolerance.

The relation between $\Delta \boldsymbol{r}$ and $\Delta \boldsymbol{d}$ is thus found by means of the $\Delta \tilde{\boldsymbol{d}}$ values. One might try to relate the residual $r$ directly to $\boldsymbol{d}$ instead of to $\tilde{d}$, but this obviously will not work as the new input for $\mathcal{S} \circ \mathcal{F}$ would be a linear combination of the previous inputs. The only new information in the input of $\mathcal{S} \circ \mathcal{F}$ would originate from numerical errors and consequently the coupling iterations would not converge. More details can be found in [27].

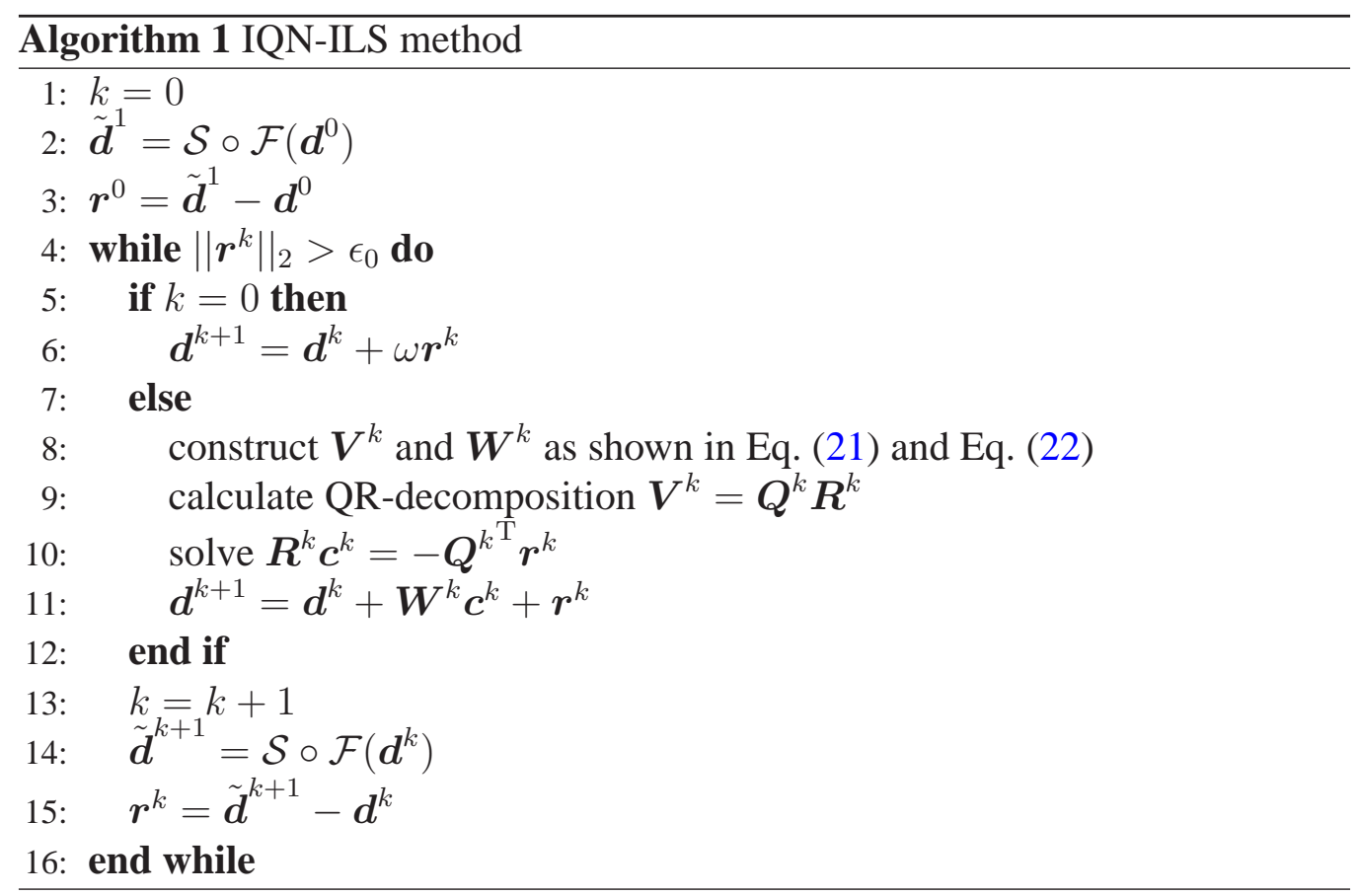




\section{Gauss-Seidel iterations with Aitken relaxation}

If the interaction between the fluid and the structure is strong then GaussSeidel iterations between the flow solver and the structural solver diverge quickly without any relaxation. However, it is difficult to determine a priori a value for the relaxation factor which will result in fast convergence of the Gauss-Seidel iterations. Aitken relaxation [30, 31] signifies that a dynamically varying scalar relaxation factor $\omega^{k}$ is used for the Gauss-Seidel iterations within a time step. The next displacement of the fluid-structure interface is calculated as

$$
\begin{aligned}
\boldsymbol{d}^{k+1} & =\boldsymbol{d}^{k}+\omega^{k} \boldsymbol{r}^{k} \\
& =\left(1-\omega^{k}\right) \boldsymbol{d}^{k}+\omega^{k} \tilde{\boldsymbol{d}}^{k+1}
\end{aligned}
$$

and consequently the next input for $\mathcal{S} \circ \mathcal{F}$ is a linear combination of the last output and the previous input. Moreover, the update of the interface's position is in the direction of the residual vector, as opposed to the update from the IQNILS method. The first relaxation in a time step is executed with the relaxation factor from the end of the previous time step, but limited to $\omega^{\max }$, so $\omega^{0}=$ $\operatorname{sign}\left(\omega^{n}\right) \min \left(\left|\omega^{n}\right|, \omega^{\max }\right)$. The value of $\omega^{k}$ is obtained as

$$
\omega^{k}=-\omega^{k-1} \frac{\left(\boldsymbol{r}^{k-1}\right)^{\mathrm{T}}\left(\boldsymbol{r}^{k}-\boldsymbol{r}^{k-1}\right)}{\left(\boldsymbol{r}^{k}-\boldsymbol{r}^{k-1}\right)^{\mathrm{T}}\left(\boldsymbol{r}^{k}-\boldsymbol{r}^{k-1}\right)} .
$$

\section{Rolling tank}

The rolling tank cases presented by Idelsohn et al. [9] are simulated to verify the coupling code and both solvers. These cases consist of a rectangular container partially filled with oil or water. This fluid interacts with a flexible structure which is clamped to either the top or bottom of the tank. The container rotates around the midpoint of its bottom and a harmonic rolling motion is imposed by an electric motor. Three different configurations are considered, namely a standing beam immersed in shallow oil (Figure 3), a standing beam immersed in deep oil (Figure 4) and a hanging beam above shallow water (Figure 5).

For this rolling tank, data from experiments and two-dimensional monolithic PFEM calculations are available [9]. The experiments have been performed with a transparent tank such that images could be taken. The displacement of the tip of the beam in the rotating reference frame of the tank has been calculated from these images with a computer programme. Special attention has been paid to the 


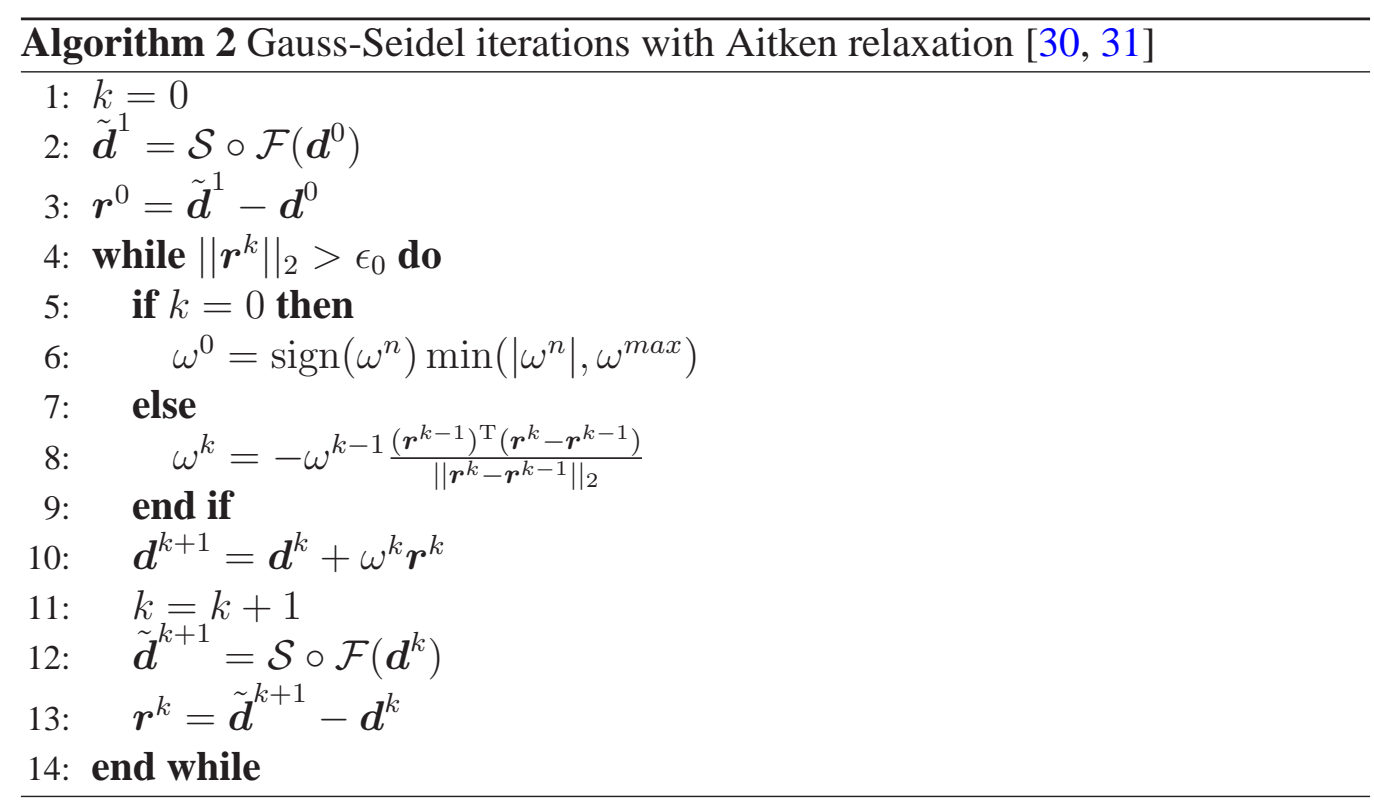

gaps between the flexible structure and the front and back of the tank such that the experiments can be considered two-dimensional.

The tank is identical in the three configurations and it is $0.609 \mathrm{~m}$ wide and $0.3445 \mathrm{~m}$ high. The shallow liquid level is $0.0574 \mathrm{~m}$ and the deep liquid level is $0.1148 \mathrm{~m}$. The elastic beam is $0.004 \mathrm{~m}$ thick and its tip coincides with the still liquid-gas interface for both the standing and the hanging beam. The top of the tank is a constant pressure boundary while all other boundaries are zero-slip walls. Each configuration has been simulated on three different grids, named coarse, medium and fine. The coarse grid for all configurations is depicted in Figure 2 and the number of degrees-of-freedom in the fluid and solid domain of the three grids is listed in Table 1. The number of degrees-of-freedom in the fluid domain changes slightly during the simulations due to remeshing.

The angular frequency of the rolling motion that is imposed on the tank corresponds to the fundamental frequency of gravitational waves in a liquid of limited depth [39], given by

$$
\omega=\sqrt{\frac{\pi g}{L} \tanh \frac{\pi H}{L}}
$$

with $H$ the height of the liquid and $L$ the width of the tank. The period of the rolling motion is thus $1.65 \mathrm{~s}$ for the shallow oil or water configuration and $1.21 \mathrm{~s}$ for the deep oil configuration. For the standing beam, the amplitude of the rolling 
motion is $4^{\circ}$ and for the hanging beam it is $2^{\circ}$. However, when the motor is started there is a transition from the rest state to the harmonic motion due to inertia and therefore the true time-angle curves [9] have been employed. The time step is $0.0025 \mathrm{~s}$ for the standing beam and $0.0010 \mathrm{~s}$ for the hanging beam, which corresponds to at least 500 time steps in one period of the rolling motion.

The properties of the liquid, gas and solid can be found in Table 2 for the three configurations. The structure is discretized with rectangular 8-node continuum finite elements with reduced integration. The plane stress approximation to the linear-elastic material law Eq. (8) is used for the isotropic material

$$
\left[\begin{array}{l}
\epsilon_{11} \\
\epsilon_{22} \\
\epsilon_{12}
\end{array}\right]=\left[\begin{array}{ccc}
1 / E & -\nu / E & 0 \\
-\nu / E & 1 / E & 0 \\
0 & 0 & 1 / G
\end{array}\right]\left[\begin{array}{l}
\sigma_{11} \\
\sigma_{22} \\
\sigma_{12}
\end{array}\right]
$$

with the shear modulus calculated as $G=\frac{E}{2(1+\nu)}$.

The shape of the deformed structure and the position of the liquid-gas interface are compared with experimental data in Figure 3 for the standing beam immersed in shallow oil, in Figure 4 for the standing beam immersed in deep oil and in Figure 5 for the hanging beam. In the numerical results, the hanging beam exhibits a slight bend near its midpoint after the impact of the water on the structure. Consequently, higher bending modes are active in the numerical model. A nonlinear material model which performs a curve fit of tension test data does not eliminate this difference in behaviour. Accurate data from bending tests might improve the result but such tests are difficult to perform on a rubber material.

For a more quantitative comparison, Figure 6 depicts the displacement of the tip of the beam parallel to the bottom of the tank (in the rotating reference frame) for the three configurations and the three grids. For all configurations, the differences between the grids are small. The agreement between the experiments and the numerical results is good, especially for the standing beam in deep oil. The measurement error is relatively large for the standing beam in shallow oil because the displacements are an order of magnitude smaller than for the standing beam in deep oil. For the standing beam in shallow oil and for the hanging beam, the difference between the partitioned results and the experiments is larger but still similar to the difference between the monolithic PFEM simulations and the experiments [9].

In each time step, the $L_{2}$-norm of the residual is reduced with three orders of magnitude with respect to its value in the first coupling iteration of the time step $\left(\epsilon_{o}=10^{-3}\left\|r^{0}\right\|_{2}\right)$. The number of IQN-ILS coupling iterations per time step 
(averaged over all time steps) is listed in Table 3 for all grids and it can be seen that the number of IQN-ILS coupling iterations is independent of the number of degrees-of-freedom. From the perspective of the IQN-ILS coupling algorithm, the hanging beam case is easiest (i.e. fewer coupling iterations per time step) because the beam comes mainly into contact with air which has a lower density than the liquid such that the added-mass effect is smaller [1, 24, 25].

All simulations are subsequently repeated using Gauss-Seidel iterations with Aitken relaxation. Also for Aitken relaxation, there is no significant influence of the number of grid points on the number of coupling iterations per time step. Especially for the standing beam, the algorithm with Aitken relaxation requires significantly more coupling iterations per time step while the IQN-ILS algorithm is only slightly faster than Aitken relaxation for the hanging beam. The larger performance gap between the IQN-ILS algorithm and Aitken relaxation for the standing beam cases compared to the hanging beam case is explained by the stability analysis in $[24,25]$. In this analysis, the error on the interface's displacement during Gauss-Seidel coupling iterations is decomposed in different components, each with its own wave number. More components in the residual vector $r$ become unstable or badly damped during the coupling iterations if the density of the fluid increases and the amplification factor of each component depends on its wave number. Because the IQN-ILS coupling algorithm has been developed based on this stability analysis, the residual vector is decomposed in components (Eq. (23)) and each component is treated differently (Eq. (26)). It has been observed during the simulations that the columns of the matrix $\boldsymbol{V}$ have a different wave number. Aitken relaxation, on the other hand, uses the same relaxation factor for the entire residual vector (Eq. (29)) and so it does not take into account that the components with different wave numbers have different amplification factors. For the hanging beam case where the beam mainly comes into contact with low density air, there are only very few unstable components in the residual vector so the decomposition of the IQN-ILS algorithm does not result in a significant benefit.

The comparison of the wall clock time of the IQN-ILS algorithm and GaussSeidel iterations with Aitken relaxation is almost identical to the comparison of the number of coupling iterations. This is due to the fact that the CPU time for the calculations of the coupling algorithms themselves is negligible with respect to the CPU time required for the solution of the discrete equations in the fluid and solid domain. The information from previous time steps could not be reused by the IQN-ILS algorithm to improve the approximation of the Jacobian's inverse and to reduce the number of coupling iterations as in [27] due to the large difference in behaviour between the time steps. 


\section{Falling composite cylinder}

Wave-energy converters that consist of several buoys, so-called "floating point absorbers", which move relative to a large floating platform are currently under development in the Sustainable Economically Efficient Wave Energy Converter (SEEWEC) project $[40,41]$. The Buldra test platform and one of the absorbers are depicted in Figure 7. A hydraulic circuit with a turbine converts the vertical motion of the absorbers into a rotary motion which is used to drive a generator. The absorbers are made of a composite material by means of filament winding. They have to meet diverse requirements and the impact of the absorber on the water surface (vertical slamming) or the impact of waves on the absorber (horizontal or breaking wave slamming) are important design aspects. The hydrodynamic impact pressure locally deforms the absorber, which will damage the composite material in time. To accelerate the design process of these wave-energy converters, the fluid-structure interaction during the impact of both a rigid and a deformable composite cylinder on a water surface is simulated numerically. Previous studies of the impact of a cylinder on a water surface ([42, 43, 44], among others) analyze metal cylinders or employ linearized calculation techniques.

The geometry for this simulation can be seen in Figure 8; only half of the cylinder is simulated due to the symmetry. The fluid domain is two-dimensional and it consists of a rectangular box around the cylinder which has an outer diameter of $d_{o}=0.3 \mathrm{~m}$. The box is $1.5 d_{o}$ wide and it extends from $2 d_{o}$ above the centre of the cylinder to $3 d_{o}$ below the centre. The cylinder's bottom is positioned $0.025 \mathrm{~m}$ above the water surface at the beginning of the fluid-structure interaction simulation. Atmospheric pressure is applied on the top boundary and symmetry is imposed on the right boundary. The remaining boundaries of the fluid domain, including the fluid-structure interface, are no-slip walls. The structural model is a three-dimensional cylinder with a thickness of $0.003 \mathrm{~m}$. It is discretized with 4-node shell elements with reduced integration but it only contains one row of elements along the axis of the cylinder. The height of the cylinder is calculated to obtain square shell elements. The nodes on the symmetry line are constrained such that they can only move vertically; the nodes on the front and back of the cylinder cannot translate along the cylinder's axis and they can only rotate around an axis parallel to the cylinder's axis. Although the three-dimensional model for the cylinder is constrained to two-dimensional motion, a three-dimensional geometry is required for the model of the composite material.

Most material parameters are concisely listed in the last column of Table 2. The composite material of the shell elements is modeled with two orthogonal 
symmetry planes for the elastic properties (orthotropic). Consequently, the plane stress approximation of the linear-elastic material law Eq. (8) is given by

$$
\left[\begin{array}{l}
\epsilon_{11} \\
\epsilon_{22} \\
\epsilon_{12}
\end{array}\right]=\left[\begin{array}{ccc}
1 / E_{1} & -\nu_{12} / E_{1} & 0 \\
-\nu_{12} / E_{1} & 1 / E_{2} & 0 \\
0 & 0 & 1 / G_{12}
\end{array}\right]\left[\begin{array}{l}
\sigma_{11} \\
\sigma_{22} \\
\sigma_{12}
\end{array}\right]
$$

in which the local material orientation is indicated with subscripts 1 to 3 so that direction 1 is aligned with the fiber and direction 3 is the normal to the surface of plane stress. The Poisson's ratio $\nu_{21}$ has been substituted by $\nu_{21}=\frac{E_{2}}{E_{1}} \nu_{12}$. For this material, the Young's moduli are $E_{1}=25.77 \cdot 10^{9} \mathrm{~N} / \mathrm{m}^{2}$ and $E_{2}=6.2519$. $10^{9} \mathrm{~N} / \mathrm{m}^{2}$, the shear modulus is $G_{12}=4.2 \cdot 10^{9} \mathrm{~N} / \mathrm{m}^{2}$ and the Poisson's ratio is $\nu_{12}=0.38$. The shear moduli $G_{13}=4.2 \cdot 10^{9} \mathrm{~N} / \mathrm{m}^{2}$ and $G_{23}=2.5 \cdot 10^{9} \mathrm{~N} / \mathrm{m}^{2}$ are used to model transverse shear deformation in the shell. The tensile $(t)$ and compressive (c) stress limit is $\sigma_{1 t}=\sigma_{1 c}=600 \cdot 10^{6} \mathrm{~N} / \mathrm{m}^{2}$ in the fiber direction and $\sigma_{2 t}=\sigma_{2 c}=60 \cdot 10^{6} \mathrm{~N} / \mathrm{m}^{2}$ in the transverse direction; the shear strength of the material is $\sigma_{12 s}=50 \cdot 10^{6} \mathrm{~N} / \mathrm{m}^{2}$.

Rayleigh damping has been added to the structure with a factor of $\alpha_{r}=5.46$ for the mass-proportional contribution to the damping and $\beta_{r}=1.41 \cdot 10^{-5}$ for the stiffness-proportional contribution. The factors $\alpha_{r}$ and $\beta_{r}$ have been calculated so that the first and second eigenmode of the cylinder have a damping ratio of 0.01 which is a typical value for multilayered cylindrical shell structures [45, 46].

During the production of the absorbers, the fibers are wound in different directions. This is modeled with a shell section that contains five layers of $0.6 \cdot 10^{-3} \mathrm{~m}$ thick. The fibers of the inner layer are perpendicular to the cylinder's axis, while the four outer layers and the axis meet alternately at an angle of $70^{\circ}$ and $-70^{\circ}$. Simpson's rule with 3 points in each layer is used for the integration through the thickness of the shell section. This material model is available in many existing structural codes but not yet in most monolithic codes for fluid-structure interaction, which stresses the benefit of partitioned simulations with black-box solvers.

The impact of the composite cylinder on the water surface has been simulated on three different grids, named coarse, medium and fine. The coarse grid is depicted in Figure 8 and the number of degrees-of-freedom in the fluid and solid domain of all grids is listed in Table 1. Again, the number of degrees-of-freedom in the fluid domain changes slightly during the simulations due to remeshing. The time step is $100 \cdot 10^{-7} \mathrm{~s}$ on the coarse grid, $50 \cdot 10^{-7} \mathrm{~s}$ on the medium grid and $25 \cdot 10^{-7} \mathrm{~s}$ on the fine grid so as to obtain a Courant number $\mathrm{C}=\frac{v_{o} \Delta t}{\Delta x}$ of 0.02 on all grids, based on the initial velocity of the cylinder $\left(v_{o}\right)$ and the height of the 
cells adjacent to the cylinder $(\Delta x)$. Because especially the impact is of interest, a short period of $0.0125 \mathrm{~s}$ is simulated on the fine grid and a longer time span of $0.0250 \mathrm{~s}$ and $0.0500 \mathrm{~s}$ is simulated on the medium and coarse grid, respectively.

The cylinder is given a downward velocity of $v_{o}=5 \mathrm{~m} / \mathrm{s}$ in the structural solver at the onset of the fluid-structure interaction calculation $(t=0)$. Consequently, the gas phase that surrounds the cylinder has to move at the same speed at the beginning of the coupled simulation; otherwise the gas in the cells adjacent to the cylinder would be accelerated from $0 \mathrm{~m} / \mathrm{s}$ to $5 \mathrm{~m} / \mathrm{s}$ in the first time step. To obtain a proper initialization of the fluid domain, the grids are constructed so that the centre of the cylinder in the fluid grid is located at $\frac{2}{3} v_{o} T$ (with $T=10^{-2} \mathrm{~s}$ ) above the centre of the cylinder in the structural grid. The fluid domain is then initialized with all velocities equal to zero at time $t=-T$. Before the beginning of the coupled calculation, 100 time steps of $10^{-4} \mathrm{~s}$ are performed with the flow solver only to step from $t=-T$ to $t=0$. During these time steps, the no-slip wall that represents the outside of the cylinder is moved downwards as a rigid body with prescribed vertical velocity

$$
v=v_{o}\left[\left(\frac{t}{T}\right)^{2}-1\right] .
$$

The gas that surrounds the cylinder is consequently accelerated from $0 \mathrm{~m} / \mathrm{s}$ to a downward velocity $v_{o}$ and the position of the centre of the cylinder is identical in the fluid and solid domain at $t=0$.

During the fluid-structure interaction calculation, the cylinder first falls through the air region and then it impacts on the water surface around $t=5 \cdot 10^{-3} \mathrm{~s}$. There is no exact time of impact because there is no exact position of the liquid-gas interface as this interface is not tracked with grid points but reconstructed from the volume fraction. The shape of the free-surface during the impact is displayed in Figure 9. These plots show that the cylinder is first compressed vertically (Figure 9(c)) and then stretched vertically (Figure 9(e)). This can also be observed in Figure 10(a) which depicts the deformation of the cylinder, defined as the difference between the initial and current value of the distance between the top and the bottom of the cylinder. The deformation is small while the cylinder is traversing the air region but it increases rapidly during the impact on the water surface. After the initial contact, the deformation oscillates with decreasing amplitude. The maximal deformation amounts to approximately $6 \%$ of the cylinder's diameter.

Figure 10(b) displays the vertical velocity at the bottom of the cylinder as a function of time. The simulation on the coarse grid has been performed with the 
flexible cylinder as described above but also with a "rigid" cylinder which has thousand times larger stiffness moduli than the flexible cylinder. At impact, the velocity at the bottom of the cylinder jumps from $-5 \mathrm{~m} / \mathrm{s}$ to $-2 \mathrm{~m} / \mathrm{s}$ for the flexible cylinder, followed by oscillations due to the interaction between the inertia in the flexible structure and in the fluid. The velocity decreases more gradually for the rigid cylinder as it barely deforms. The vertical force on the entire cylinder is shown in Figure 10(c) and the peak at impact is much higher for the rigid cylinder, as expected. As the force is proportional to the acceleration and thus to the second time derivative of the displacement, it is much more difficult to have a smooth evolution of the force than a smooth evolution of the displacement. Consequently, few authors show stresses or forces as a function of time.

Figure 10 shows that the solution of the different grids is very close to each other, especially for the medium and fine grid. The maximal deformation is almost identical on all grids but there is a small difference in the time of impact between the coarse grid on one hand and the medium and fine grid on the other hand, as can be seen in Figure 10(b) and Figure 10(c). Because decreasing the time step with a factor two on the coarse grid does not yield significant improvement, it can be concluded that the difference is mainly due to the grid.

Figure 11 depicts the pressure relative to the atmospheric pressure on three different segments of the fluid-structure interface as a function of time for the flexible and the rigid cylinder. The delay between the peaks at the different locations can clearly be observed and the amplitude of the peak decreases as one moves away from the bottom of the cylinder. The peak of the pressure at the bottom of the cylinder is $182 \cdot 10^{3} \mathrm{~N} / \mathrm{m}^{2}$ for the flexible cylinder and $279 \cdot 10^{3} \mathrm{~N} / \mathrm{m}^{2}$ for the rigid cylinder, which proves that the fluid-structure interaction must be taken into account during the design process to avoid a too strong and therefore a too costly product. The minimal pressure during the oscillations in $t \in[0,0.0125] \mathrm{s}$ is $18 \cdot 10^{3} \mathrm{~N} / \mathrm{m}^{2}$ below atmospheric pressure for the flexible cylinder and $3 \cdot 10^{3} \mathrm{~N} / \mathrm{m}^{2}$ below atmospheric pressure for the rigid cylinder. The absolute pressure is in both cases higher than the vapour pressure of pure water which is $2338 \mathrm{~N} / \mathrm{m}^{2}$ at $293 \mathrm{~K}$ and thus no cavitation occurs.

The damage to the composite material due to the impact is assessed with the Tsai-Wu failure criterion in plane stress condition [47] which requires that

$$
I_{F}=F_{1} \sigma_{11}+F_{2} \sigma_{22}+F_{11} \sigma_{11}^{2}+F_{22} \sigma_{22}^{2}+F_{66} \sigma_{12}^{2}+2 F_{12} \sigma_{11} \sigma_{22}<1
$$


with

$$
\begin{aligned}
F_{1} & =\frac{1}{\sigma_{1 t}}+\frac{1}{\sigma_{1 c}} \\
F_{2} & =\frac{1}{\sigma_{2 t}}+\frac{1}{\sigma_{2 c}} \\
F_{11} & =\frac{-1}{\sigma_{1 t} \sigma_{1 c}} \\
F_{22} & =\frac{-1}{\sigma_{2 t} \sigma_{2 c}} \\
F_{66} & =\frac{1}{\sigma_{12 s}^{2}} \\
F_{12} & =f \sqrt{F_{11} F_{22}} .
\end{aligned}
$$

The tensile and compressive strength in the fiber direction and in the transverse direction are given above. Often, the coupling coefficient $f$ is set to 0 so that $F_{12}$ disappears. The value of $I_{F}$ is analyzed in all time steps near the impact and in all layers of the composite material. Its maximal value is 0.25 so well below the limit.

In each time step, the $L_{2}$-norm of the residual is reduced with three orders of magnitude with respect to its value in the first coupling iteration $\left(\epsilon_{o}=10^{-3}\left\|\boldsymbol{r}^{0}\right\|_{2}\right)$. The number of coupling iterations per time step is displayed in Figure 12 for the simulation on the coarse grid. About 5 IQN-ILS iterations per time step are required during the first 500 time steps while the cylinder is falling through air. However, approximately 11 IQN-ILS iterations per time step are necessary to reach convergence in the time steps in which there is contact between the cylinder and the water. This difference illustrates the effect of the fluid density on the stability of the coupling iterations $[1,24,25]$. The number of coupling iterations per time step (averaged over all time steps and over all time steps between $t=0$ and $t=0.0125 \mathrm{~s}$ ) is similar for all grids as can be seen in Table 3. This proves that the performance of the IQN-ILS coupling algorithm is independent of the number of degrees-of-freedom. For comparison, the simulation on the coarse grid are also performed using Gauss-Seidel iterations with Aitken relaxation which requires almost twice as many coupling iterations to reach the same convergence tolerance. The number of coupling iterations per time step is limited to 20. As in the previous section, reuse of information from previous time steps to improve the approximation of the Jacobian's inverse and consequently reduce the number of coupling iterations as used in [27] does not function well in this particular case due to the large difference in behaviour between the time steps during the impact. 


\section{Conclusion}

The numerical results demonstrate that the interaction between free surface flow and an elastic structure can be simulated in a partitioned way using the IQNILS coupling algorithm, even for cases with strong interaction due to the incompressibility of the fluid. Gauss-Seidel iterations with Aitken relaxation can also be used but this requires more coupling iterations. Both coupling algorithms treat the flow solver and the structural solver as a black box, meaning that existing solvers can be reused without modifications. Consequently, the partitioned blackbox approach allows to combine complex models which are readily available in many flow solvers and structural solvers but not yet in monolithic fluid-structure interaction solvers.

The rolling tank cases of Idelsohn et al. [9] are used as a verification and the partitioned simulations correspond well with experimental data. Subsequently, the impact of a flexible composite cylinder on a water surface is simulated to assess the effect of slamming on the absorbers of floating wave-energy converters. The impact of the flexible cylinder is significantly different from the impact of a rigid cylinder, which stresses the need for fluid-structure interaction calculations in the design process. Grid refinement has been performed for all calculations and the coupling algorithm performs similarly on each grid.

In future research, the influence of a turbulence model in FSI simulations has to be investigated as this could be relevant for some cases with free surface flow, for example for the hanging beam above shallow water. In general, the hanging beam case is the one with the highest discrepancies. Future studies to reduce these discrepancies might be low Reynolds experiments, further assessment of the gap influence in the experiments, determination of the uncertainty on the mechanical properties of the solid and sensitivity of the simulations to such uncertainties.

The experimental and numerical data for Figure 6 and Figure 10 can be found online on http://www.FSI.UGent.be/files/fsi_free_surface.zip or http://www.FSI.UGent.be/files/fsi_free_surface.tar.gz.

\section{Acknowledgments}

J. Degroote gratefully acknowledges a Ph.D. fellowship of the Research Foundation - Flanders (FWO). 


\section{References}

[1] P. Causin, J.-F. Gerbeau, F. Nobile, Added-mass effect in the design of partitioned algorithms for fluid-structure problems, Computer Methods in Applied Mechanics and Engineering 194 (42-44) (2005) 4506-4527.

[2] R. van Loon, P. Anderson, F. van de Vosse, A fluid-structure interaction method with solid-rigid contact for heart valve dynamics, Journal of Computational Physics 217 (2006) 806-823.

[3] K. Dumont, J. Vierendeels, R. Kaminsky, G. Van Nooten, P. Verdonck, D. Bluestein, Comparison of the hemodynamic and thrombogenic performance of two bileaflet mechanical heart valves using a CFD/FSI model, Journal of Biomechanical Engineering - Transactions of the ASME 129 (4) (2007) 558-565.

[4] P. Le Tallec, J. Mouro, Fluid structure interaction with large structural displacements, Computer Methods in Applied Mechanics and Engineering 190 (2001) 3039-3067.

[5] T. Tezduyar, S. Sathe, K. Stein, Solution techniques for the fully discretized equations in computation of fluid-structure interactions with the space-time formulations, Computer Methods in Applied Mechanics and Engineering 195 (41-43) (2006) 5743-5753.

[6] E. Walhorn, A. Kölke, B. Hübner, D. Dinkler, Fluid-structure coupling within a monolithic model involving free surface flows, Computers \& Structures 83 (25-26) (2005) 2100-2111.

[7] C. Antoci, M. Gallati, S. Sibilla, Numerical simulation of fluid-structure interaction by SPH, Computers \& Structures 85 (11-14) (2007) 879-890.

[8] S. Idelsohn, J. Marti, A. Limache, E. Oñate, Unified Lagrangian formulation for elastic solids and incompressible fluids: Application to fluid-structure interaction problems via the PFEM, Computer Methods in Applied Mechanics and Engineering 197 (2008) 1762-1776.

[9] S. Idelsohn, J. Marti, A. Souto-Iglesias, E. Oñate, Interaction between an elastic structure and free-surface flows: experimental versus numerical comparisons using the PFEM, Computational Mechanics 43 (2008) 125-132. 
[10] S. Potapov, B. Maurel, A. Combescure, J. Fabis, Modeling accidental-type fluid-structure interaction problems with the SPH method, Computers \& Structures 87 (11-12) (2009) 721-734.

[11] J. Glimm, J. Grove, X. Li, W. Oh, D. Sharp, A critical analysis of RayleighTaylor growth rates, Journal of Computational Physics 169 (2001) 652-677.

[12] G. Ryskin, L. Leal, Numerical solution of free-boundary problems in fluid mechanics. Part 2. Bouyancy-driven motion of a gas bubble through a quiescent liquid, Journal of Fluid Mechanics 148 (1984) 19-35.

[13] J. Degroote, P. Bruggeman, J. Vierendeels, A coupling algorithm for partitioned solvers applied to bubble and droplet dynamics, Computers \& Fluids 38 (3) (2009) 613-624.

[14] C. Hirt, B. Nichols, Volume of fluid method for the dynamics of free boundaries, Journal of Computational Physics 39 (1981) 201-225.

[15] W. Rider, D. Kothe, Reconstructing volume tracking, Journal of Computational Physics 141 (1998) 112-152.

[16] S. Osher, R. Fedkiw, Level set methods: an overview and some recent results, Journal of Computational Physics 169 (2001) 463-502.

[17] E. Oñate, S. Idelsohn, F. Del Pin, R. Aubry, The particle finite element method. An overview, International Journal of Computational Methods 1 (2004) 267-307.

[18] J. Monaghan, Simulating free surface flows with SPH, Journal of Computational Physics 110 (2) (1994) 399-406.

[19] D. Rothman, S. Zaleski, Lattice-gas models of phase separation: interfaces, phase transitions and multiphase flow, Reviews of Modern Physics 66 (1994) 1417-1479.

[20] A. Colagrossi, M. Landrini, Numerical simulation of interfacial flows by smoothed particle hydrodynamics, Journal of Computational Physics 191 (2) (2003) 448-475.

[21] M. Heil, An efficient solver for the fully coupled solution of largedisplacement fluid-structure interaction problems, Computer Methods in Applied Mechanics and Engineering 193 (2004) 1-23. 
[22] C. Felippa, K. Park, C. Farhat, Partitioned analysis of coupled mechanical systems, Computer Methods in Applied Mechanics and Engineering 190 (2001) 3247-3270.

[23] C. Förster, W. Wall, E. Ramm, Artificial added mass instabilities in sequential staggered coupling of nonlinear structures and incompressible viscous flows, Computer Methods in Applied Mechanics and Engineering 196 (7) (2007) 1278-1293.

[24] J. Degroote, P. Bruggeman, R. Haelterman, J. Vierendeels, Stability of a coupling technique for partitioned solvers in FSI applications, Computers \& Structures 86 (23-24) (2008) 2224-2234.

[25] J. Degroote, S. Annerel, J. Vierendeels, Stability analysis of Gauss-Seidel iterations in a partitioned simulation of fluid-structure interaction, Computers \& Structures In press.

[26] J. Vierendeels, L. Lanoye, J. Degroote, P. Verdonck, Implicit coupling of partitioned fluid-structure interaction problems with reduced order models, Computers \& Structures 85 (11-14) (2007) 970-976.

[27] J. Degroote, K.-J. Bathe, J. Vierendeels, Performance of a new partitioned procedure versus a monolithic procedure in fluid-structure interaction, Computers \& Structures 87 (11-12) (2009) 793-801.

[28] C. Michler, E. van Brummelen, R. de Borst, An interface Newton-Krylov solver for fluid-structure interaction, International Journal for Numerical Methods in Fluids 47 (10-11) (2005) 1189-1195.

[29] H. Matthies, R. Niekamp, J. Steindorf, Algorithms for strong coupling procedures, Computer Methods in Applied Mechanics and Engineering 195 (2006) 2028-2049.

[30] D. Mok, W. Wall, Partitioned analysis schemes for the transient interaction of incompressible flows and nonlinear flexible structures, in: K. Schweizerhof, K. Wall, W.A. Bletzinger (Eds.), Trends in computational structural mechanics, CIMNE, Barcelona, 2001.

[31] U. Küttler, W. Wall, Fixed-point fluid-structure interaction solvers with dynamic relaxation, Computational Mechanics 43 (2008) 61-72. 
[32] S. Patankar, Numerical Heat Transfer and Fluid Flow, Hemisphere, Washington, DC, USA, 1980.

[33] D. Youngs, Numerical Methods for Fluid Dynamics, chap. Time-Dependent Multi-Material Flow with Large Fluid Distortion, Academic Press, New York, NY, USA, 273-285, editors: Morton, K.W. and Baines, M.J., 1982.

[34] H. Hilber, T. Hughes, R. Taylor, Improved numerical dissipation for time integration algorithms in structural dynamics, Earthquake Engineering \& Structural Dynamics 5 (3) (1977) 283-292.

[35] C. Farhat, M. Lesoinne, P. Le Tallec, Load and motion transfer algorithms for fluid/structure interaction problems with non-matching discrete interfaces: momentum and energy conservation, optimal discretization and application to aeroelasticity, Computer Methods in Applied Mechanics and Engineering 157 (1998) 95-114.

[36] A. de Boer, A. van Zuijlen, H. Bijl, Review of coupling methods for nonmatching meshes, Computer Methods in Applied Mechanics and Engineering 196 (2007) 1515-1525.

[37] R. Haelterman, J. Degroote, D. Van Heule, J. Vierendeels, The quasi-Newton least squares method: a new and fast secant method analyzed for linear systems, SIAM Journal on numerical analysis 47 (3) (2009) 2347-2368.

[38] G. H. Golub, C. F. V. Loan, Matrix computations, Johns Hopkins University Press, Baltimore, MD, USA, 3rd edn., 1996.

[39] H. Lamb, Hydrodynamics, chap. 9. Surface Waves, Cambridge University Press, 6 edn., 365-369, 1932.

[40] G. De Backer, M. Vantorre, R. Banasiak, J. De Rouck, C. Beels, H. Verhaeghe, Performance of a point absorber heaving with respect to a floating platform, in: 7th European Wave and Tidal Energy Conference, Porto, Portugal, 1, 2007.

[41] C. Blommaert, W. Van Paepegem, J. Degrieck, Design of composite material for cost effective large scale production of components for floating offshore structures, Plastics rubber and composites 38 (2) (2009) 146-152. 
[42] M.-C. Lin, L.-D. Shieh, Flow visualization and pressure characteristics of a cylinder for water impact, Applied Ocean Research 19 (1997) 101-112.

[43] M. Ionina, A. Korobkin, Water impact on cylindrical shells, in: R. Beck, W. Schultz (Eds.), International Workshop on Water Waves and Floating Bodies, Port Huron, MI, USA, 44-47, 1999.

[44] H. Sun, O. Faltinsen, Water impact of horizontal circular cylinders and cylindrical shells, Applied Ocean Research 28 (2006) 299-311.

[45] N. Alam, N. Asnani, Vibration and damping analysis of a multilayered cylindrical shell, Part I: Theoretical analysis, AIAA Journal 22 (6) (1984) 803810.

[46] N. Alam, N. Asnani, Vibration and damping analysis of a multilayered cylindrical shell, Part II: Numerical results, AIAA Journal 22 (7) (1984) 975-981.

[47] S. Tsai, E. Wu, General theory of strength for anisotropic materials, Journal of Composite Materials 5 (1971) 58-80. 


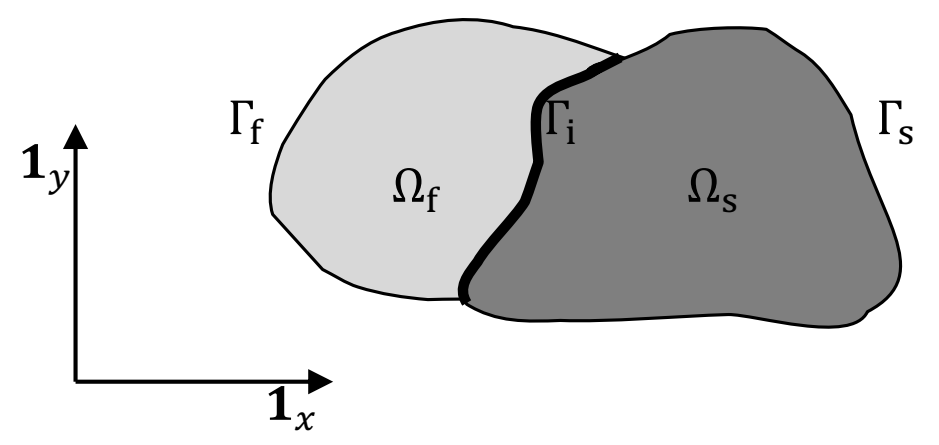

Figure 1: The fluid subdomain $\Omega_{f}$, the solid subdomain $\Omega_{s}$, their boundaries $\Gamma_{f}$ and $\Gamma_{s}$ and the fluid-structure interface $\Gamma_{i}$. 


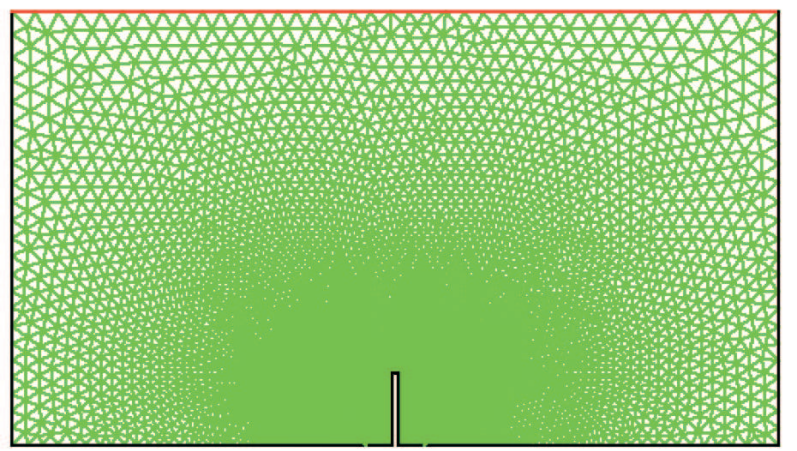

(a)

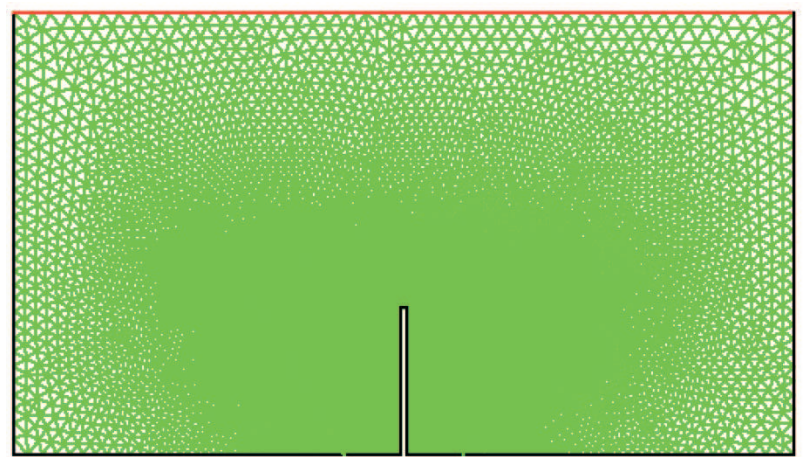

(b)

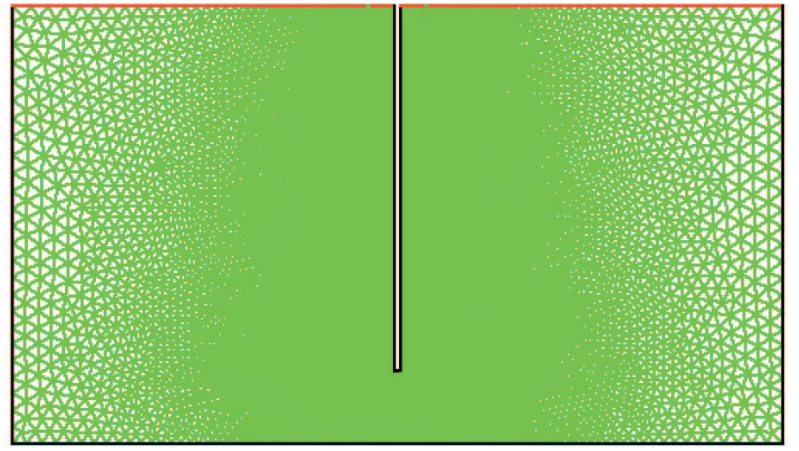

(c)

Figure 2: The coarse grid for the simulation of the rolling tank with (a) a standing beam in shallow oil, (b) a standing beam in deep oil and (c) a hanging beam above shallow water. A constant pressure is imposed on the red boundary, green is the fluid domain and black represents a no-slip wall or the structural domain. 

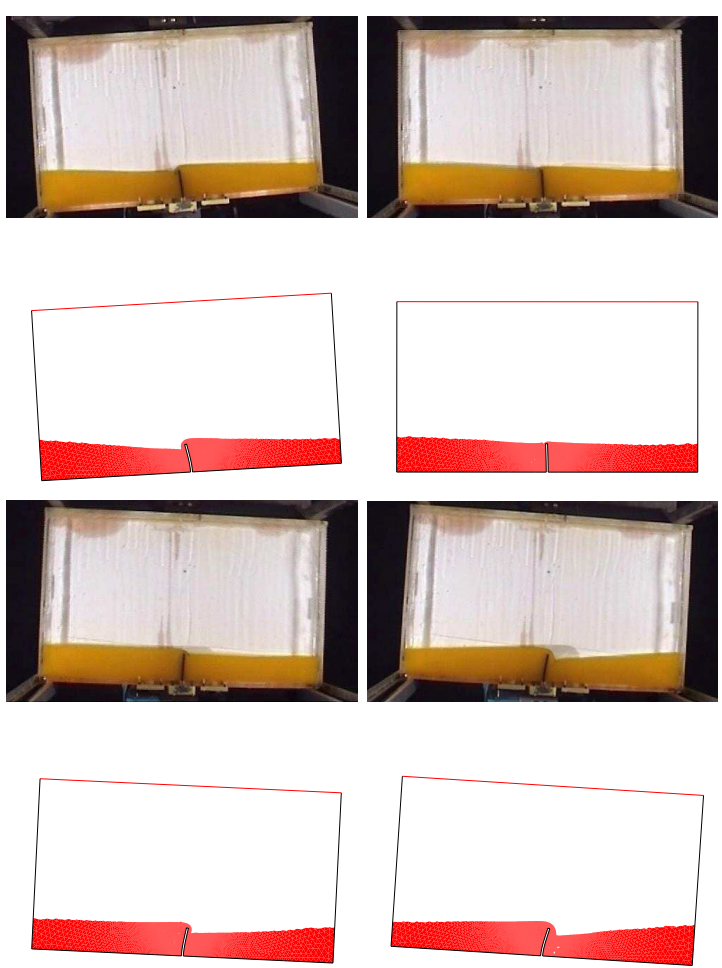

Figure 3: The comparison between the experimental data and the numerical results on the fine grid of the rolling tank with a standing beam in shallow oil after $0.92 \mathrm{~s}, 1.20 \mathrm{~s}, 1.40 \mathrm{~s}$ and $1.68 \mathrm{~s}$. 

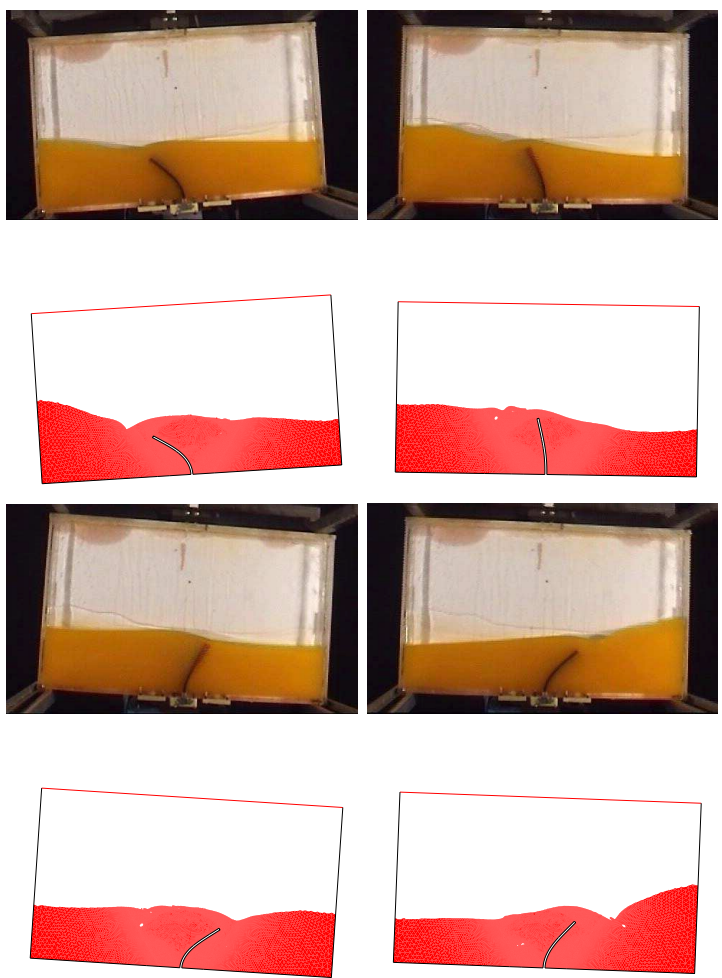

Figure 4: The comparison between the experimental data and the numerical results on the fine grid of the rolling tank with a standing beam in deep oil after $1.84 \mathrm{~s}, 2.12 \mathrm{~s}, 2.32 \mathrm{~s}$ and $2.56 \mathrm{~s}$. 

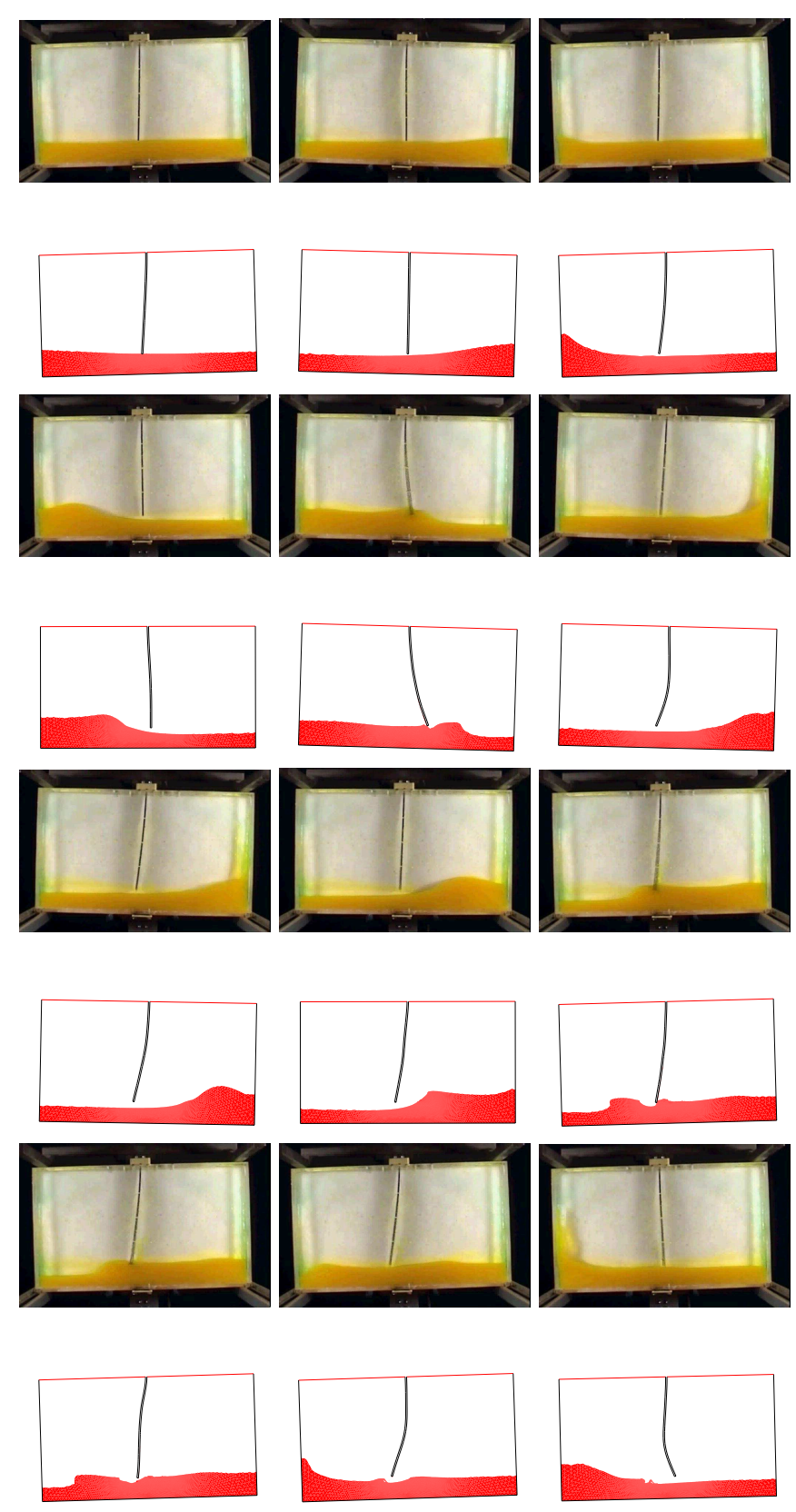

Figure 5: The comparison between the experimental data and the numerical results on the fine grid of the rolling tank with a hanging beam above shallow water after $0.76 \mathrm{~s}, 1.64 \mathrm{~s}, 2.40 \mathrm{~s}, 2.68 \mathrm{~s}$, $2.96 \mathrm{~s}, 3.32 \mathrm{~s}, 3.40 \mathrm{~s}, 3.56 \mathrm{~s}, 3.80 \mathrm{~s}, 3.84 \mathrm{~s}, 4.00 \mathrm{~s}$ and $4.16 \mathrm{~s}$. 


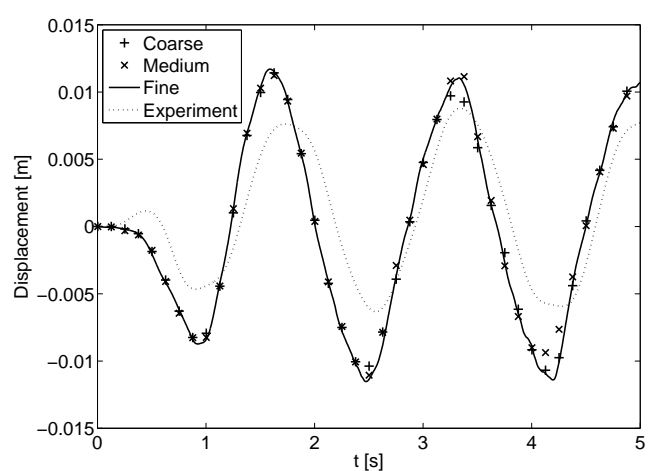

(a)

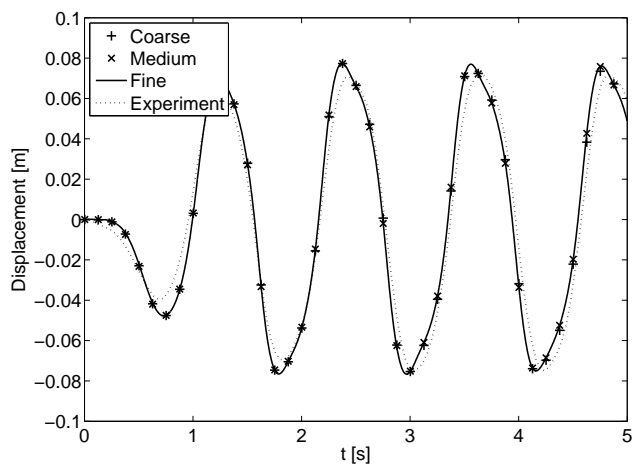

(b)

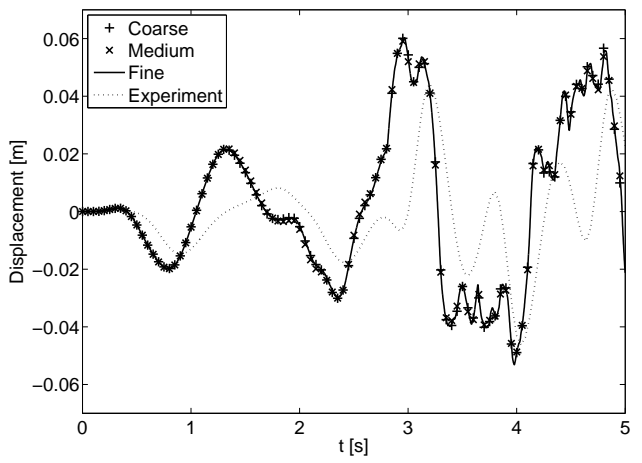

(c)

Figure 6: The displacement of the tip of the beam parallel to the bottom of the tank (in the rotating reference frame) for the simulation of the rolling tank with (a) a standing beam in shallow oil, (b) a standing beam in deep oil and (c) a hanging beam above shallow water. 


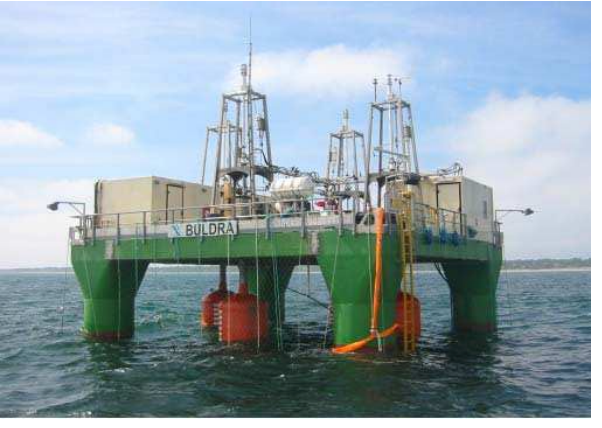

(a)

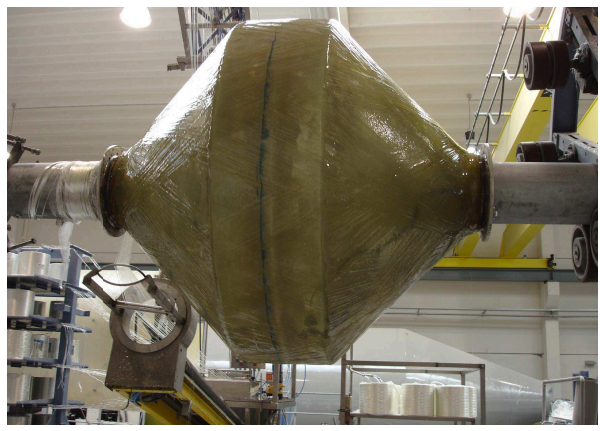

(b)

Figure 7: (a) The Buldra test platform of the SEEWEC project and (b) a composite point absorber produced by means of filament winding. 


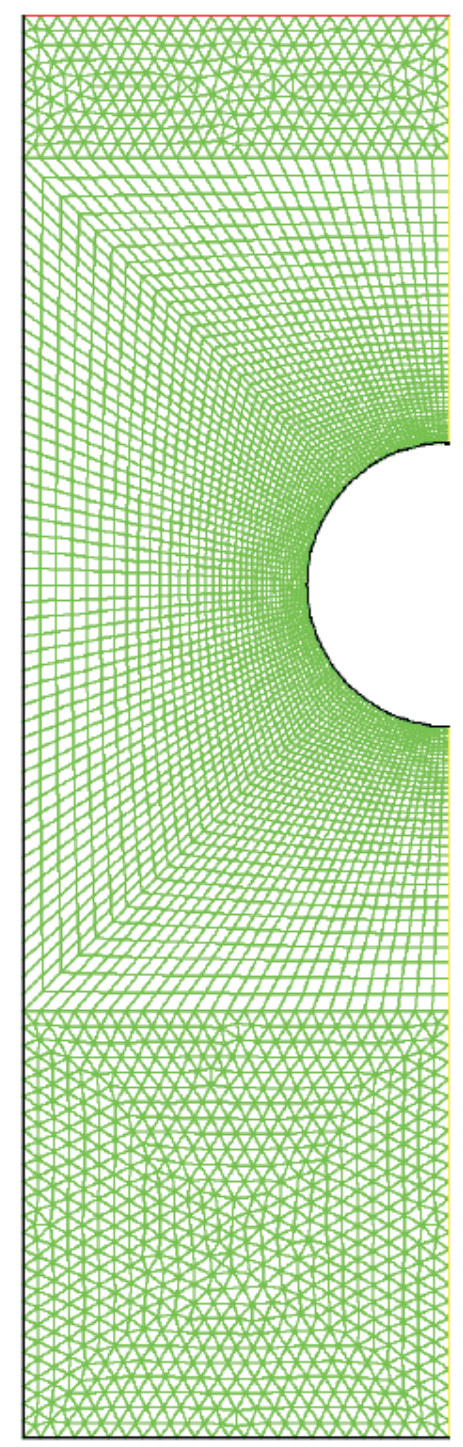

Figure 8: The coarse grid for the simulation of the falling cylinder. A constant pressure is imposed on the red boundary, yellow means a symmetry boundary, green is the fluid domain and black represents a no-slip wall or the structural domain. 


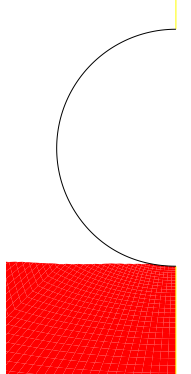

(a)

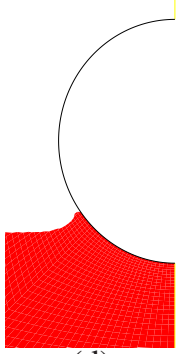

(d)

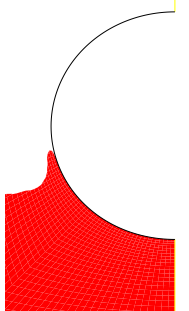

(g)

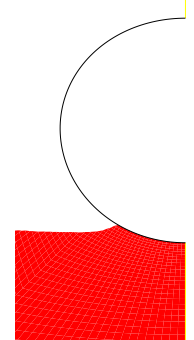

(b)

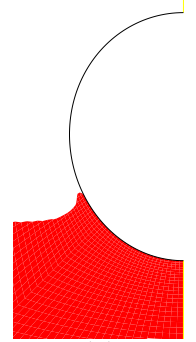

(e)

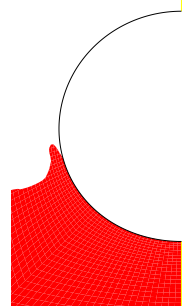

(h)

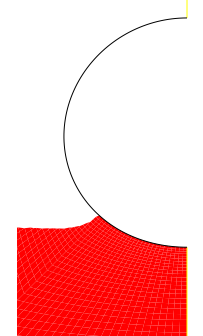

(c)

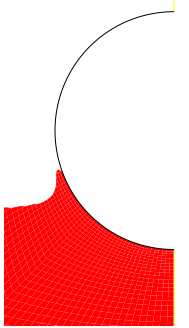

(f)

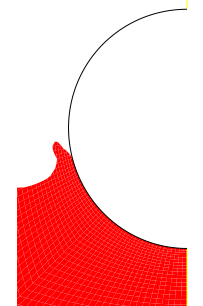

(i)

Figure 9: The shape of the water surface on the coarse grid of the falling flexible cylinder after (a) $0.005 \mathrm{~s}$, (b) $0.010 \mathrm{~s}$, (c) $0.015 \mathrm{~s}$, (d) $0.020 \mathrm{~s}$, (e) $0.025 \mathrm{~s}$, (f) $0.030 \mathrm{~s}$, (g) $0.035 \mathrm{~s}$, (h) $0.040 \mathrm{~s}$ and (i) $0.045 \mathrm{~s}$. 


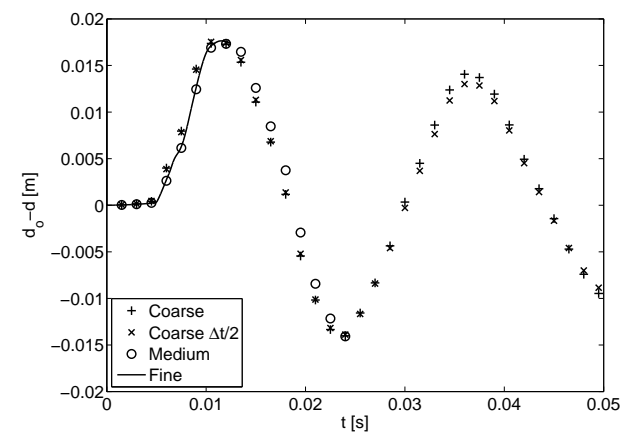

(a)

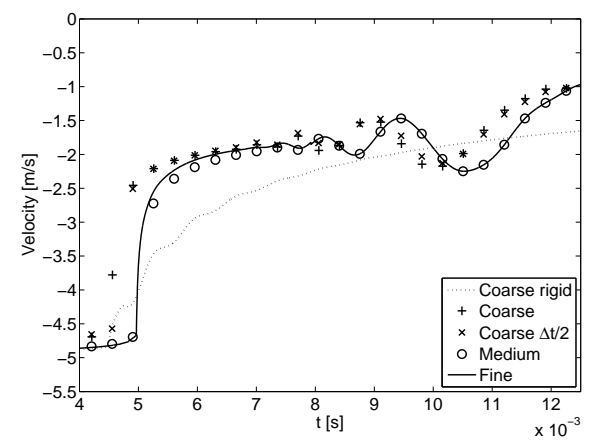

(b)

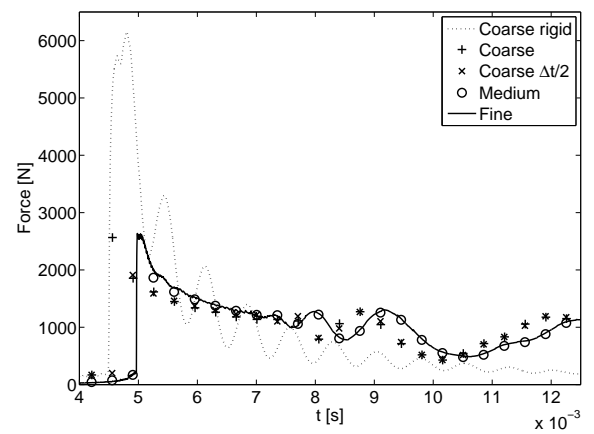

(c)

Figure 10: (a) The deformation of the flexible cylinder as a function of time for the falling cylinder. The deformation is defined as the initial distance between the top and bottom of the cylinder $\left(d_{o}\right)$ minus the current distance between the top and bottom $(d)$. (b) The vertical velocity at the bottom of the rigid and flexible cylinder as a function of time. (c) The vertical force on the entire rigid and flexible cylinder as a function of time. 


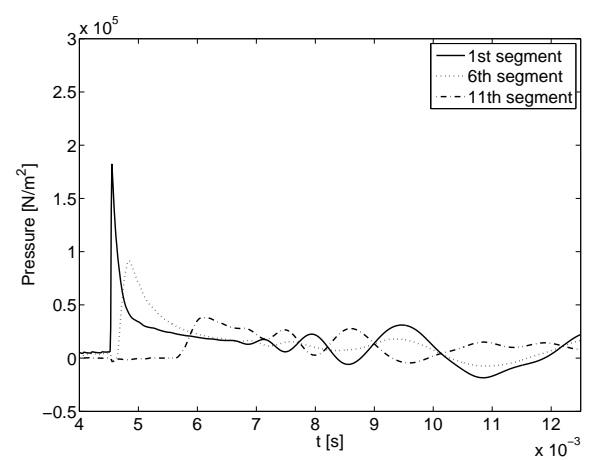

(a)

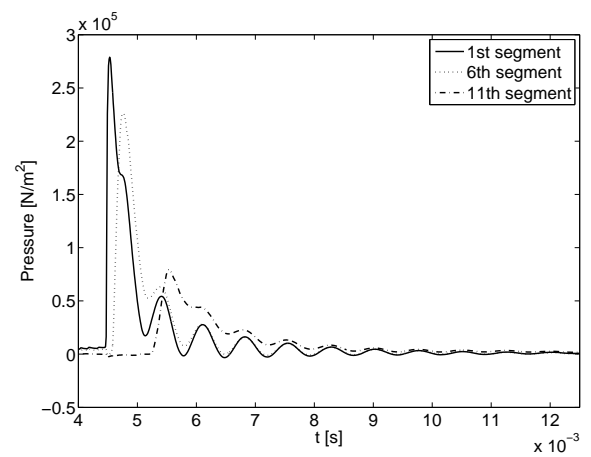

(b)

Figure 11: The pressure relative to the atmospheric pressure on the 1st, 6th and 11th segment of the fluid-structure interface (counting from the bottom, 100 segments in total) as a function of time for (a) a flexible cylinder and (b) a rigid cylinder with the coarse grid. 


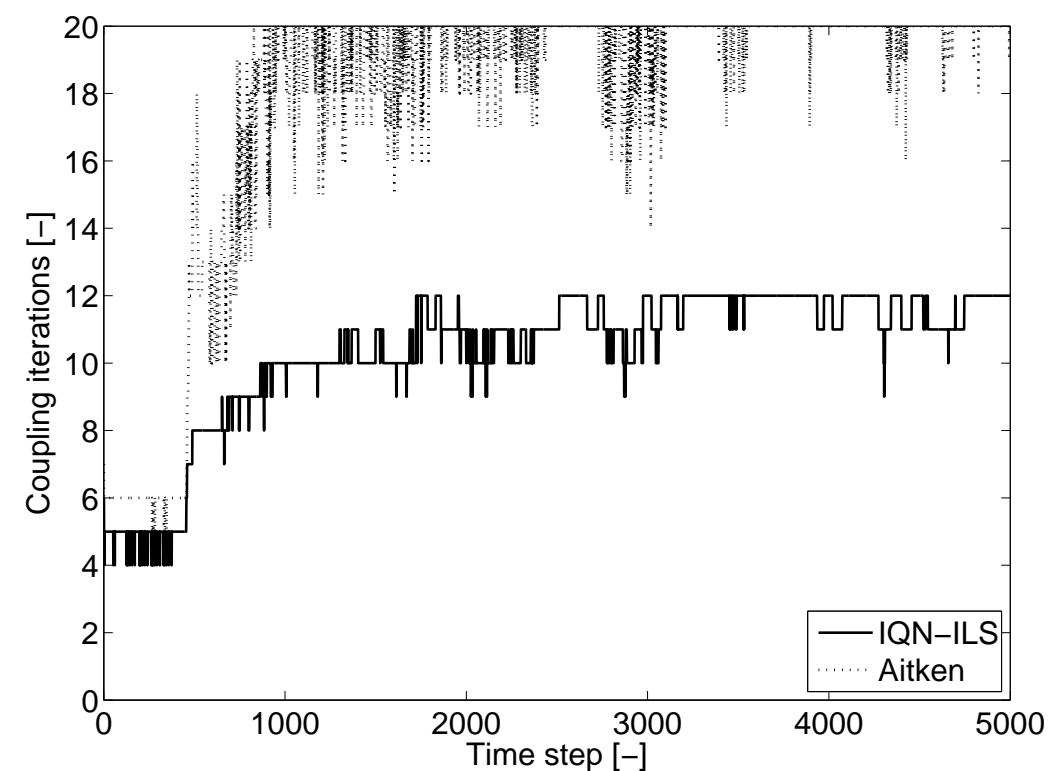

Figure 12: The number of coupling iterations per time step of the simulation of the falling flexible cylinder with the coarse grid. 


\begin{tabular}{|l|c|c|c|c|}
\cline { 2 - 5 } \multicolumn{1}{c|}{} & $\begin{array}{c}\text { Standing beam } \\
\text { shallow oil }\end{array}$ & $\begin{array}{c}\text { Standing beam } \\
\text { deep oil }\end{array}$ & $\begin{array}{c}\text { Hanging beam } \\
\text { shallow water }\end{array}$ & $\begin{array}{c}\text { Falling } \\
\text { cylinder }\end{array}$ \\
\hline Coarse & $51160+581$ & $97840+1141$ & $127888+2821$ & $22416+1212$ \\
Medium & $81016+1229$ & $171120+2429$ & $233080+6029$ & $87532+2412$ \\
Fine & $118448+2117$ & $254216+4197$ & $372688+10437$ & $354552+4812$ \\
\hline
\end{tabular}

Table 1: The number of degrees-of-freedom in the different grids for the simulations of the rolling tank and the falling cylinder. The number in front of the plus sign refers to the fluid domain, the number behind it to the solid domain. 


\begin{tabular}{|c|c|c|c|c|c|}
\cline { 2 - 6 } \multicolumn{2}{l|}{} & $\begin{array}{c}\text { Standing beam } \\
\text { shallow oil }\end{array}$ & $\begin{array}{c}\text { Standing beam } \\
\text { deep oil }\end{array}$ & $\begin{array}{c}\text { Hanging beam } \\
\text { shallow water }\end{array}$ & $\begin{array}{c}\text { Falling } \\
\text { cylinder }\end{array}$ \\
\hline \multirow{2}{*}{ Liquid } & $\rho\left[\mathrm{kg} / \mathrm{m}^{3}\right]$ & 917 & 917 & 998.2 & 998.2 \\
& $\mu[\mathrm{Pa} \cdot \mathrm{s}]$ & 0.04585 & 0.04585 & 0.001003 & 0.001003 \\
\hline \multirow{2}{*}{ Gas } & $\rho\left[\mathrm{kg} / \mathrm{m}^{3}\right]$ & 1.225 & 1.225 & 1.225 & 1.225 \\
& $\mu[\mathrm{Pa} \cdot \mathrm{s}]$ & $1.79 \cdot 10^{-5}$ & $1.79 \cdot 10^{-5}$ & $1.79 \cdot 10^{-5}$ & $1.79 \cdot 10^{-5}$ \\
\hline \multirow{3}{*}{ Solid } & $\rho\left[\mathrm{kg} / \mathrm{m}^{3}\right]$ & 1100 & 1100 & 1900 & 1900 \\
& $E\left[\mathrm{~N} / \mathrm{m}^{2}\right]$ & $6 \cdot 10^{6}$ & $6 \cdot 10^{6}$ & $4 \cdot 10^{6}$ & - \\
& $\nu[-$ & 0.49 & 0.49 & 0.49 & - \\
\hline
\end{tabular}

Table 2: The material properties for the simulations of the rolling tank and the falling cylinder. 


\begin{tabular}{|l|c|c|c|c|c|c|c|c|}
\cline { 2 - 9 } \multicolumn{1}{c|}{} & \multicolumn{2}{c|}{$\begin{array}{c}\text { Standing beam } \\
\text { shallow oil }\end{array}$} & \multicolumn{2}{c|}{$\begin{array}{c}\text { Standing beam } \\
\text { deep oil }\end{array}$} & \multicolumn{2}{c|}{$\begin{array}{c}\text { Hanging beam } \\
\text { shallow water }\end{array}$} & \multicolumn{2}{c|}{$\begin{array}{c}\text { Falling } \\
\text { cylinder }\end{array}$} \\
\cline { 2 - 9 } & IQN-ILS & Aitken & IQN-ILS & Aitken & IQN-ILS & Aitken & IQN-ILS & Aitken \\
\hline Coarse & 7.16 & 11.93 & 8.90 & 15.16 & 4.54 & 5.34 & $10.4(7.6)$ & $17.9(12.6)$ \\
Medium & 7.32 & 12.11 & 8.95 & 15.40 & 4.53 & 5.14 & $9.5(7.6)$ & \\
Fine & 7.62 & 12.53 & 8.96 & 15.30 & 4.53 & 4.92 & 8.1 & \\
\hline
\end{tabular}

Table 3: The number of coupling iterations per time step (averaged over all time steps and between brackets averaged over all time steps between $t=0$ and $t=0.0125 \mathrm{~s}$ ) for the simulations of the rolling tank and the falling cylinder. 